\title{
Spatial Besov Regularity for Stochastic Partial Differential Equations on Lipschitz Domains
}

\author{
Petru A. Cioica, Stephan Dahlke, Stefan Kinzel, Felix Lindner, \\ Thorsten RaAsch, Klaus Ritter, René L. Schilling
}

\begin{abstract}
We use the scale of Besov spaces $B_{\tau, \tau}^{\alpha}(\mathcal{O}), \alpha>0,1 / \tau=\alpha / d+1 / p, p$ fixed, to study the spatial regularity of the solutions of linear parabolic stochastic partial differential equations on bounded Lipschitz domains $\mathcal{O} \subset \mathbb{R}^{d}$. The Besov smoothness determines the order of convergence that can be achieved by nonlinear approximation schemes. The proofs are based on a combination of weighted Sobolev estimates and characterizations of Besov spaces by wavelet expansions.
\end{abstract}

Keywords: Stochastic partial differential equation, Besov space, Lipschitz domain, wavelet, weighted Sobolev space, nonlinear approximation, adaptive numerical scheme

Mathematics Subject Classification (2010): 60H15, Secondary: 46E35, 65C30

\section{Introduction}

In this paper, the spatial Besov regularity of the solutions of linear stochastic evolution equations on bounded Lipschitz domains is studied. We combine regularity results by KIM [30] on stochastic partial differential equations (SPDEs, for short) on Lipschitz domains in terms of weighted Sobolev spaces with methods used in DAHLKe, DeVore [13], where the Besov regularity of (deterministic) elliptic equations on Lipschitz domains is investigated. Our considerations are motivated by the question whether adaptive and other nonlinear approximation methods for the solutions of SPDEs on Lipschitz domains pay off in the sense that they yield better convergence rates than uniform methods. Thus referring to a

This work has been supported by the Deutsche Forschungsgemeinschaft (DFG, grants DA 360/13-1, RI 599/4-1, SCHI 419/5-1) and a doctoral scholarship of the Philipps-Universität Marburg. 
numerical theme and combining concepts and methods from different areas and scientific communities, the article is addressed to readers of both worlds: stochastic analysis and numerical analysis. Therefore, we give a rather detailed account in the first part of the paper, emphasizing conceptual and notational clarity.

Our setting is as follows. On a finite interval $[0, T] \subset[0, \infty)$ let $\left(w_{t}^{\kappa}\right)_{t \in[0, T]}, \quad \kappa \in \mathbb{N}=$ $\{1,2, \ldots\}$, be independent, one-dimensional standard Brownian motions with respect to a filtration $\left(\mathcal{F}_{t}\right)_{t \in[0, T]}$ of $\sigma$-algebras on a complete probability space $(\Omega, \mathcal{F}, \mathbb{P})$. Throughout the paper we assume that $\left(\mathcal{F}_{t}\right)_{t \in[0, T]}$ is normal, i.e. the filtration satisfies the usual hypotheses, see, e.g. [18, Section 3.3.]. Let $\mathcal{O} \subset \mathbb{R}^{d}$ be a bounded Lipschitz domain. We consider the model equation

$$
d u=\sum_{\mu, \nu=1}^{d} a^{\mu \nu} u_{x_{\mu} x_{\nu}} d t+\sum_{\kappa=1}^{\infty} g^{\kappa} d w_{t}^{\kappa}, \quad u(0, \cdot)=u_{0},
$$

for $t \in[0, T]$ and $x \in \mathcal{O}$. Here $d u$ is Itô's stochastic differential with respect to $t$, $\left(a^{\mu \nu}\right)_{1<\mu, \nu<d} \in \mathbb{R}^{d \times d}$ is a strictly positive definite, symmetric matrix and the coefficients $g^{\kappa}, \kappa \in \mathbb{N}$, are random functions depending on $t$ and $x$ such that the mappings $\Omega \times[0, T] \ni$ $(\omega, t) \mapsto g^{\kappa}(\omega, t, \cdot)$ are predictable processes with values in certain function spaces. For details see Section 2.3 .

Equation (1) is understood in a weak or distributional sense, i.e. $u$ is a solution of (1), if for all smooth and compactly supported test functions $\varphi \in C_{0}^{\infty}(\mathcal{O})$ the equality

$$
\langle u(t, \cdot), \varphi\rangle=\left\langle u_{0}, \varphi\right\rangle+\sum_{\mu, \nu=1}^{d} \int_{0}^{t}\left\langle a^{\mu \nu} u_{x_{\mu} x_{\nu}}(s, \cdot), \varphi\right\rangle d s+\sum_{\kappa=1}^{\infty} \int_{0}^{t}\left\langle g^{\kappa}(s, \cdot), \varphi\right\rangle d w_{s}^{\kappa}
$$

holds for all $t \in[0, T] \mathbb{P}$-almost surely. Here and throughout the paper we write $\langle u, \varphi\rangle$ for the application of a distribution $u \in \mathcal{D}^{\prime}(\mathcal{O})$ to a test function $\varphi \in C_{0}^{\infty}(\mathcal{O})$. The existence and uniqueness of solutions of equation (11), respectively equation (3) below, within certain classes $\mathfrak{H}_{p, \theta}^{\gamma}(\mathcal{O}, T)$ of stochastic processes has been shown in [30]; see also the earlier papers by KRYLOV, Lototsky and Kim, e.g. [29], [31], 32], 35]. Roughly speaking, the classes $\mathfrak{H}_{p, \theta}^{\gamma}(\mathcal{O}, T)$ are $L_{p}$-spaces of functions on $\Omega \times[0, T]$ with values in weighted Sobolev spaces $H_{p, \theta-p}^{\gamma}(\mathcal{O})$ that can be regarded as generalizations of the classical Sobolev spaces with zero Dirichlet boundary condition. Again we refer to Section 2.3 for precise definitions. Let us remark that in Examples 17, 18 and 19, illustrating our Besov regularity result in Section 3, the solution of equation (1) in the class $\mathfrak{H}_{2, \theta}^{\gamma}(\mathcal{O}, T)$ coincides with the unique weak solution with zero Dirichlet boundary condition in the sense of DA PRATO, ZABCZYK [18], and hence can be represented by the well known stochastic variation-of-constants formula

$$
u(t, \cdot)=e^{t A} u_{0}+\int_{0}^{t} e^{(t-s) A} G(s) d W_{s}, \quad t \in[0, T] .
$$

Here $\left(e^{t A}\right)_{t \geq 0}$ is the semigroup of contractions on $L_{2}(\mathcal{O})$ generated by the partial differential operator $A=\sum_{\mu, \nu=1}^{d} a^{\mu \nu} \frac{\partial^{2}}{\partial x_{\mu} \partial x_{\nu}}$ with zero Dirichlet boundary condition considered as an 
unbounded operator on $L_{2}(\mathcal{O}),(G(t))_{t \in[0, T]}$ is an operator-valued process and $\left(W_{t}\right)_{t \in[0, T]}$ is a cylindrical Wiener process on $\ell_{2}(\mathbb{N})$, see Remarks 13 and 14 in Section 2.3 .

As already mentioned, our motivation to study the Besov regularity of SPDEs is the theme of nonlinear approximation of the solution processes. For deterministic settings, a detailed overview of nonlinear approximation and an exposition of the characterization of its efficiency in terms of the Besov smoothness of the target functions can be found in DeVore [21], see also Cohen [8, Chapters 3 and 4]. Let us consider an example of approximation by wavelets in $L_{p}(\mathcal{O})$, the $L_{p}$-space of real-valued functions on $\mathcal{O}, p \in$ $(1, \infty)$. To this end, let $\left\{\psi_{\lambda}: \lambda \in \nabla\right\}$ be a wavelet basis on $\mathcal{O}$ and let $f \in L_{p}(\mathcal{O})$ be a target function which we want to approximate by functions $f_{N} \in L_{p}(\mathcal{O})$ belonging to certain approximation spaces $S_{N}$, where $N$ is the number of parameters used to describe the elements of $S_{N}$. We specify the index set of the wavelet basis by writing $\nabla=\bigcup_{j \geq j_{0}-1} \nabla_{j}$; the wavelets $\psi_{\lambda}, \lambda \in \nabla_{j}, j \geq j_{0}$, are those at scale levels $j \geq j_{0}$ respectively, and $\psi_{\lambda}, \lambda \in \nabla_{j_{0}-1}$, are the scaling functions at the coarsest level $j_{0} \in \mathbb{Z}$. In the case of uniform wavelet approximation up to a highest scale level $j_{0}-1+n, n \in \mathbb{N}$, the approximation spaces are

$$
S_{N}=S_{N(n)}=\left\{\sum_{j=j_{0}-1}^{j_{0}-1+n} \sum_{\lambda \in \nabla_{j}} c_{\lambda} \psi_{\lambda}: c_{\lambda} \in \mathbb{R}, \lambda \in \nabla_{j}, j \in\left\{j_{0}-1, \ldots, j_{0}-1+n\right\}\right\},
$$

where $N=N(n)=\left|\bigcup_{j=j_{0}-1}^{j_{0}-1+n} \nabla_{j}\right| \in \mathbb{N}$ is the cardinality of the set of all indices up to scale level $j_{0}-1+n$. Let $e_{N}(f)=\inf _{f_{N} \in S_{N}}\left\|f-f_{N}\right\|_{L_{p}(\mathcal{O})}$ be the corresponding approximation error measured in $L_{p}(\mathcal{O})$. It is well known that-under certain technical assumptions on the wavelet basis - the decay rate of $e_{N}(f)$ is linked to the $L_{p}$-Sobolev smoothness of the target function. More precisely, there exists an upper bound $r \in \mathbb{N}$ depending on the wavelet basis such that, for all $s \in[0, r]$,

$$
f \in W_{p}^{s}(\mathcal{O}) \quad \Longrightarrow \quad e_{N}(f) \leq C \cdot N^{-s / d}, N=N(n), n \in \mathbb{N},
$$

for some constant $C>0$ which does not depend on $N$. The fractional order Sobolev spaces $W_{p}^{s}(\mathcal{O})$ are defined in the next section. One can also show the converse

$$
\exists C>0 \forall n \in \mathbb{N}: e_{N}(f) \leq C \cdot N^{-s / d}, N=N(n) \quad \Longrightarrow \quad f \in W_{p}^{s^{\prime}}(\mathcal{O}), s^{\prime}<s .
$$

If we consider instead best $N$-term approximation as a form of nonlinear approximation, the approximation spaces are

$$
\Sigma_{N}=\left\{\sum_{\lambda \in \Lambda} c_{\lambda} \psi_{\lambda}: \Lambda \subset \nabla,|\Lambda| \leq N, c_{\lambda} \in \mathbb{R}, \lambda \in \Lambda\right\},
$$

$N \in \mathbb{N}$, and in this case the decay rate of the error $\sigma_{N}(f):=\inf _{f_{N} \in \Sigma_{N}}\left\|f-f_{N}\right\|_{L_{p}(\mathcal{O})}$ is governed by the smoothness of $f$ measured in certain $L_{\tau}(\mathcal{O})$-norms, $\tau<p$, which are weaker than the $L_{p}(\mathcal{O})$-norm: For all $\alpha \in[0, r]$,

$$
f \in B_{\tau, \tau}^{\alpha}(\mathcal{O}), \quad \frac{1}{\tau}=\frac{\alpha}{d}+\frac{1}{p} \Longrightarrow \sigma_{N}(f) \leq C \cdot N^{-\alpha / d}, N \in \mathbb{N},
$$




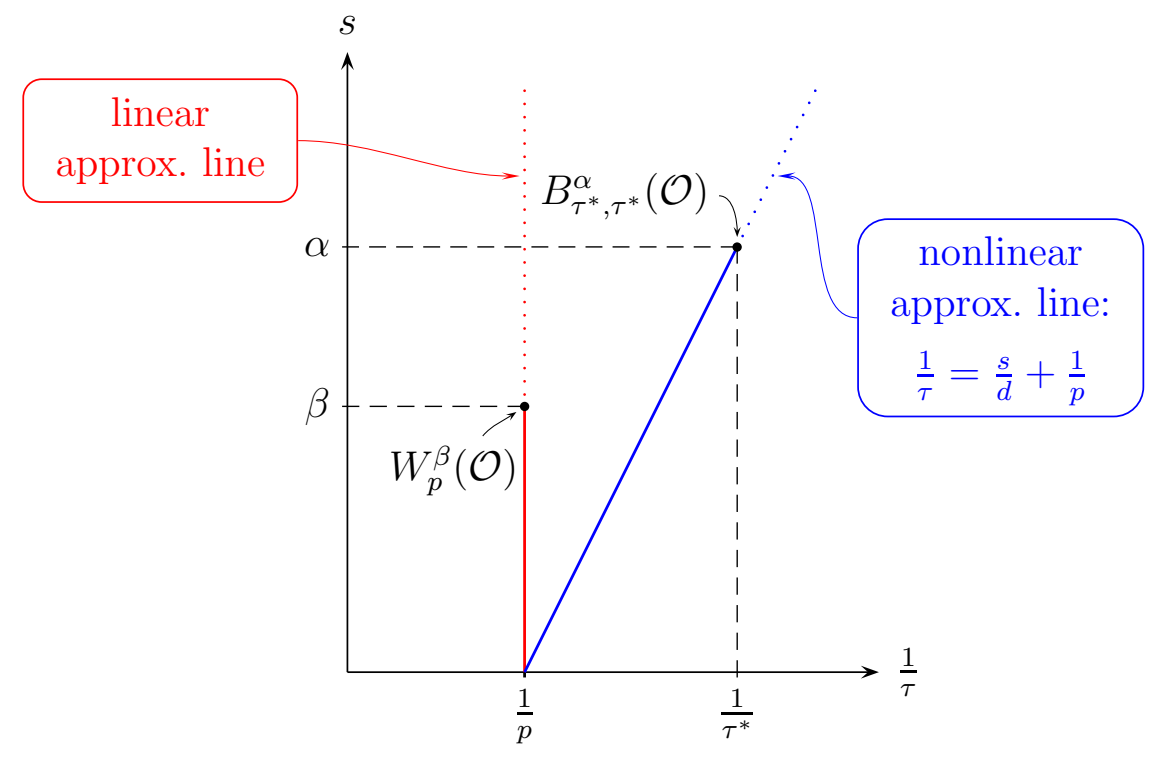

Figure 1: Linear vs. nonlinear approximation

illustrated in a DEVORE-TRIEBEL diagram.

$B_{\tau, \tau}^{\alpha}(\mathcal{O})$ being a Besov space as defined in Section 2.2. Therefore, if the target function $f$ belongs to $B_{\tau^{*}, \tau^{*}}^{\alpha}(\mathcal{O}), 1 / \tau^{*}=\alpha / d+1 / p$, for some $\alpha \in[0, r]$, and if in addition $\beta:=\sup \{s \in$ $\left.\mathbb{R}: f \in W_{p}^{s}(\mathcal{O})\right\}<\alpha$, then the convergence rate of uniform wavelet approximations is inferior to the convergence rate of the best $N$-term wavelet approximation. The latter can be considered as a benchmark for the convergence rate of adaptive numerical algorithms, see [9], [10], 12]. This situation is illustrated in Figure 1, where each point $(1 / \tau, s)$ represents the smoothness spaces of functions with " $s$ derivatives in $L_{\tau}(\mathcal{O})$ ". Note that the nonlinear approximation line $\left\{(1 / \tau, s) \in[0, \infty)^{2}: 1 / \tau=s / d+1 / p\right\}$ is also the Sobolev embedding line. For bounded domains, all spaces left to this line as well as the spaces $B_{\tau, \tau}^{s}(\mathcal{O})$ on the line are continuously embedded in $L_{p}(\mathcal{O})$.

Let us return to equation (11) and assume that the solution $u=u(\omega, t, x),(\omega, t, x) \in$ $\Omega \times[0, T] \times \mathcal{O}$, vanishes on the boundary $\partial \mathcal{O}$, satisfying a zero Dirichlet boundary condition. It is clear that the smoothness of $x \mapsto u(\omega, t, x)$ depends on the smoothness of the mappings $x \mapsto g^{\kappa}(\omega, t, x), \kappa \in \mathbb{N}$. However, even if the spatial smoothness of the $g^{\kappa}$ is high, the Sobolev smoothness of $x \mapsto u(\omega, t, x)$ can be additionally limited by singularities of the spatial derivatives of $u$ at the boundary of $\mathcal{O}$, due to the zero Dirichlet boundary condition and the shape of the domain. Such corner singularities are typical examples for the fact that the spatial $L_{p}$-Sobolev regularity of $u$ may be exceeded by the regularity in the scale of Besov spaces $B_{\tau, \tau}^{\alpha}(\mathcal{O}), 1 / \tau=\alpha / d+1 / p$. In this paper, we present a result on the spatial Besov regularity of the solution $u$ to equation (1) which has the following structure: If

$$
u \in L_{p}\left(\Omega \times[0, T], \mathcal{P}, \mathbb{P} \otimes \lambda ; W_{p}^{s}(\mathcal{O})\right)
$$


and if the functions $g^{\kappa}, \kappa \in \mathbb{N}$, are sufficiently regular, then

$$
u \in L_{\tau}\left(\Omega \times[0, T], \mathcal{P}, \mathbb{P} \otimes \lambda ; B_{\tau, \tau}^{\alpha}(\mathcal{O})\right)
$$

for certain $\alpha>s$ and $1 / \tau=\alpha / d+1 / p$. Here $\mathcal{P}$ is the predictable $\sigma$-algebra w.r.t. the filtration $\left(\mathcal{F}_{t}\right)_{t \in[0, T]}$ and $\lambda$ denotes Lebesgue measure on $[0, T]$. This result is important for the theoretical foundation of adaptive numerical methods for the approximation of $u$. The proof is based on a wavelet expansion of an extension of $\mathcal{O} \ni x \mapsto u(\omega, t, x)$ to $\mathbb{R}^{d}$, which allows us to estimate the $B_{\tau, \tau}^{\alpha}(\mathcal{O})$-norm in terms of the wavelet coefficients. We apply a strategy similar to the one used in DAHLKE, DEVore [13], where the Besov regularity of (deterministic) elliptic equations on Lipschitz domains is investigated with the help of an estimate of weighted Sobolev norms of harmonic functions. Our substitute for the latter is an estimate of weighted Sobolev norms of the solution of equation (1) provided by KIM [30].

There exists an extensive literature on the Besov regularity of SPDEs. In general, however, the assumptions on the domain and the scale of parameters considered do not fit into our setting. To mention an example, the semigroup approach to SPDEs of DA PRATO, ZABCZYK [18], which is placed in a Hilbert space framework, has been generalized to M-type 2 Banach spaces by BRzEźNiAK [4], [5], for the purpose of gaining better Hölder regularity results. Roughly speaking, the operator $A$ appearing in equation (2) is considered as the generator of a semigroup on $L_{p}(\mathcal{O})$ for some $p \geq 2$, and the stochastic integral in (2) is considered as an stochastic integral in an interpolation space $X$ between $L_{p}(\mathcal{O})$ and $D(A) \subset L_{p}(\mathcal{O})$, the domain of $A$, realizing a zero Dirichlet boundary condition. If $\partial \mathcal{O}$ is sufficiently smooth, then $D(A)=W_{p}^{2}(\mathcal{O}) \cap \stackrel{\circ}{W}_{p}^{1}(\mathcal{O})$ and $X \subseteq B_{p, 2}^{s}(\mathcal{O})$ for some $s \in[0,2]$. In this situation, the Sobolev embedding theorem leads to Hölder regularity results, and these results become better for large $p$. With the help of a theory of stochastic integration in wider classes of Banach spaces, this approach has been generalized in the works of VAN NEERVEN, Weis, Dettweiler and VeraAr, see, e.g. [20], [37], 38], [39], compare also Brzeźniak, VAN NEERVEN [6]. In contrast to these works the problem considered here is of a different nature. Firstly, we are explicitly interested in domains with non-smooth boundary. For polygonal non-convex domains, it is well known that $W_{2}^{2}(\mathcal{O}) \cap \stackrel{\circ}{W}_{2}^{1}(\mathcal{O}) \varsubsetneqq D(A)$, where $D(A):=\left\{u \in \stackrel{\circ}{W}_{2}^{1}(\mathcal{O}): A u \in L_{2}(\mathcal{O})\right\}, A=\Delta=\sum_{\mu=1}^{d} \frac{\partial^{2}}{\partial x_{\mu}^{2}}$, see Grisvard [24], 25], and for more general Lipschitz domains see JERISOn, KENIG [28]. Secondly, we are interested in the special scale $B_{\tau, \tau}^{\alpha}(\mathcal{O}), 1 / \tau=\alpha / d+1 / p, \tau>0, p$ fixed, including in particular spaces which are no Banach spaces but quasi-Banach spaces. The parameter $\tau$ decreases if $\alpha$ increases and $B_{\tau, \tau}^{\alpha}(\mathcal{O})$ fails to be a Banach space for $\tau<1$. While our methods work in this setting, any direct approach requires (at least!) a fully-fledged theory of stochastic integration in quasi-Banach spaces which is not yet available.

Let us emphasize that our result can be extended to more general linear equations of 
the type

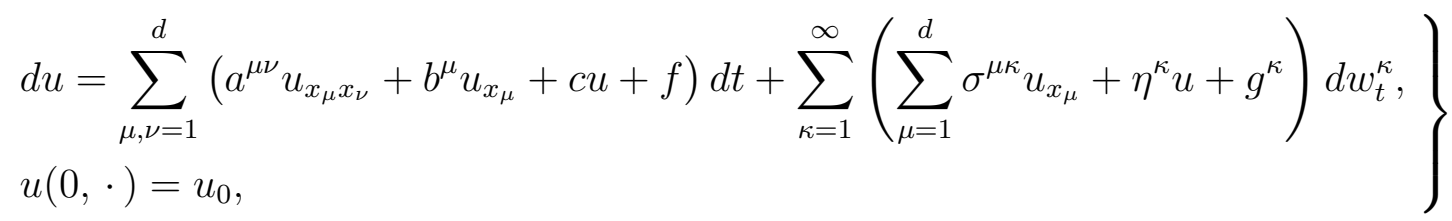

including, in particular, the case of multiplicative noise. Here the coefficients $a^{\mu \nu}, b^{\mu}, c$, $\sigma^{\mu \kappa}, \eta^{\kappa}$ and the free terms $f$ and $g^{\kappa}$ are random functions depending on $t$ and $x$. This extension is possible because one of our main tools, the weighted Sobolev norm estimate of Corollary 12 in Section 2.3, holds for equations of type (11) as well as for equations of type (3). Since this mainly adds notational complications, we will focus on equation (1) and refer to Appendix $\mathrm{B}$ for a short account of how to treat equations of type (3).

The paper is organized as follows: In Section 2 we collect the notations, definitions and preliminary results needed later on. Some general notations are introduced in Section 2.1. Section 2.2 provides the necessary facts on Besov spaces and wavelet decompositions. In Section 2.3 a short introduction to the general $L_{p}$-theory of SPDEs on Lipschitz domains due to KIM [30] is given, including definitions of the already mentioned spaces $H_{p, \theta-p}^{\gamma}(\mathcal{O}), \mathfrak{H}_{p, \theta}^{\gamma}(\mathcal{O}, T)$. Finally, in Section 3 the Besov regularity result (Theorem 15) is stated and proved, and some concrete examples for an application of the result are given.

\section{Preliminaries}

\subsection{Some notations and conventions}

In this and the next subsection $\mathcal{O} \subseteq \mathbb{R}^{d}$ can be an arbitrary (not necessarily bounded) Lipschitz domain. A domain is called Lipschitz if each point on the boundary $\partial \mathcal{O}$ has a neighbourhood whose intersection with the boundary - after relabeling and reorienting the coordinate axes if necessary - is the graph of a Lipschitz function

By $\mathcal{D}^{\prime}(\mathcal{O})$ we denote the space of Schwartz distributions on $\mathcal{O}$. If not explicitly stated otherwise, all function spaces or spaces of distributions are meant to be spaces of real-valued functions or distributions. If $f \in \mathcal{D}^{\prime}(\mathcal{O})$ is a generalized function and $\alpha=\left(\alpha_{1}, \ldots, \alpha_{d}\right) \in$ $\mathbb{N}_{0}^{d}$ is a multi-index, we write $D^{\alpha} f=\frac{\partial^{|\alpha|} f}{\partial x_{1}^{\alpha_{1}} \ldots \partial x_{d}^{\alpha_{d}}}$ for the corresponding derivative w.r.t. $x=\left(x_{1}, \ldots, x_{d}\right) \in \mathcal{O}$, where $|\alpha|=\alpha_{1}+\ldots+\alpha_{d}$. As in equations (11) and (3) we also use the notation $f_{x_{\mu} x_{\nu}}=\frac{\partial^{2} f}{\partial x_{\mu} \partial x_{\nu}}, f_{x_{\mu}}=\frac{\partial f}{\partial x_{\mu}}$. For $m \in \mathbb{N}_{0}, D^{m} f=\left\{D^{\alpha} f:|\alpha|=m\right\}$ is the set of all $m$-th order derivatives of $f$ which is identified with an $\mathbb{R}\left(\begin{array}{c}d+m-1 \\ m\end{array}\right)$-valued distribution. Given $p \in[1, \infty)$ and $m \in \mathbb{N}_{0}, W_{p}^{m}(\mathcal{O})$ denotes the classical Sobolev space consisting of all (equivalence classes of) measurable functions $f: \mathcal{O} \rightarrow \mathbb{R}$ such that $\|f\|_{W_{p}^{m}(\mathcal{O})}=\|f\|_{L_{p}(\mathcal{O})}+$ $|f|_{W_{p}^{m}(\mathcal{O})}=\left(\int_{\mathcal{O}}|f(x)|^{p} d x\right)^{1 / p}+\sum_{|\alpha|=m}\left(\int_{\mathcal{O}}\left|D^{\alpha} f(x)\right|^{p} d x\right)^{1 / p}$ is finite. For $p \in(1, \infty)$ and $s \in(m, m+1), m \in \mathbb{N}_{0}$, we define the fractional order Sobolev space $W_{p}^{s}(\mathcal{O})$ to be the Besov space $B_{p, p}^{s}(\mathcal{O})$ introduced in the next subsection. (This scale of fractional order 
Sobolev spaces can also be obtained by real interpolation of $W_{p}^{n}(\mathcal{O}), n \in \mathbb{N}_{0}$. One can show that $W_{2}^{n}(\mathcal{O})=B_{2,2}^{n}(\mathcal{O})$ for all $n \in \mathbb{N}$ and $W_{p}^{n}(\mathcal{O}) \subset B_{p, p}^{n}(\mathcal{O})$ for all $n \in \mathbb{N}, p>2$, see, e.g. Triebel [44, Remark 2.3.3/4 and Theorem 4.6.1.(b)] together with Dispa [23].) Given any countable index set $\mathcal{J}$, the space of $p$-summable sequences indexed by $\mathcal{J}$ is denoted by $\ell_{p}=\ell_{p}(\mathcal{J})$ and $|\cdot|_{\ell_{p}}$ is the respective norm. Usually we have $\ell_{p}=\ell_{p}(\mathbb{N})$ but, for instance we may also use the notation $\left|D^{m} f(x)\right|_{\ell_{p}}^{p}=\sum_{|\alpha|=m}\left|D^{\alpha} f(x)\right|^{p}$ for $f \in W_{p}^{m}(\mathcal{O})$.

Given a distribution $f \in D^{\prime}(\mathcal{O})$ and a smooth and compactly supported test function $\varphi \in C_{0}^{\infty}(\mathcal{O})$, we write $\langle f, \varphi\rangle$ for the application of $f$ to $\varphi$. If $H$ is a Hilbert space, then $\langle\cdot, \cdot\rangle_{H}$ denotes the inner product in $H$. Given another Hilbert space $U$, we denote by $L_{(\mathrm{HS})}(H, U)$ and $L_{(\text {nuc })}(H, U)$ the spaces of Hilbert-Schmidt operators and nuclear operators from $H$ to $U$ respectively, see, e.g. Pietsch [46, Sections 6 and 15] or Da Prato, ZABCZYK [18, Appendix C] for definitions. We also abbreviate $L_{(\mathrm{HS})}(H)=L_{(\mathrm{HS})}(H, H)$ and $L_{\text {(nuc) }}(H)=L_{\text {(nuc) }}(H, H) \cdot \mathcal{M}_{T}^{2, c}\left(H,\left(\mathcal{F}_{t}\right)\right)$ is the space of continuous, square integrable, $H$-valued martingales with respect to the filtration $\left(\mathcal{F}_{t}\right)_{t \in[0, T]}$. For $\Omega \times[0, T]$ we use the shorthand notation $\Omega_{T}$ and

$$
\left.\left.\mathcal{P}=\sigma(\{] s, t] \times F_{s}: 0 \leq s<t \leq T, F_{s} \in \mathcal{F}_{s}\right\} \cup\left\{\{0\} \times F_{0}: F_{0} \in \mathcal{F}_{0}\right\}\right)
$$

is the predictable $\sigma$-algebra. $\mathbb{P} \otimes \lambda$ is the product measure of the probability measure $\mathbb{P}$ on $(\Omega, \mathcal{F})$ and Lebesgue measure $\lambda$ on $([0, T], \mathcal{B}([0, T]))$, where $\mathcal{B}([0, T])$ denotes the Borel $\sigma$-algebra on $[0, T]$. Given any measure space $(A, \mathcal{A}, m)$, any (quasi-)normed space $B$ with (quasi-)norm $\|\cdot\|_{B}$ and any summability index $p>0$, we denote by $L_{p}(A, \mathcal{A}, m ; B)$ the $L_{p^{-}}$ space of all strongly measurable functions $u: A \rightarrow B$ whose (quasi-)norm $\|u\|_{L_{p}(A, \mathcal{A}, m ; B)}:=$ $\left(\int_{A}\|u(z)\|_{B}^{p} m(d z)\right)^{1 / p}$ is finite.

All equalities of random variables or random (generalized) functions appearing in this paper are meant to be $\mathbb{P}$-almost sure equalities. Throughout the paper, $C$ denotes a positive constant which may change its value from line to line.

\subsection{Besov spaces and wavelet decompositions}

In this section we give the definition of Besov spaces and describe their characterization in terms of wavelets. Our standard reference in this context is the monograph of CoHEN [8].

For a function $f: \mathcal{O} \rightarrow \mathbb{R}$ and a natural number $n \in \mathbb{N}$ let

$$
\Delta_{h}^{n} f(x):=\prod_{i=0}^{n} \mathbb{1}_{\mathcal{O}}(x+i h) \cdot \sum_{j=0}^{n}\left(\begin{array}{l}
n \\
j
\end{array}\right)(-1)^{n-j} f(x+j h)
$$

be the $n$-th difference of $f$ with step $h \in \mathbb{R}^{d}$. For $p \in(0, \infty)$ the modulus of smoothness is given by

$$
\omega^{n}(t, f)_{p}:=\sup _{|h|<t}\left\|\Delta_{h}^{n} f\right\|_{L_{p}(\mathcal{O})}, \quad t>0 .
$$

One approach to introduce Besov spaces is the following. 
Definition 1. Let $s, p, q \in(0, \infty)$ and $n \in \mathbb{N}$ with $n>s$. Then $B_{p, q}^{s}(\mathcal{O})$ is the collection of all functions $f \in L_{p}(\mathcal{O})$ such that

$$
|f|_{B_{p, q}^{s}(\mathcal{O})}:=\left(\int_{0}^{\infty}\left[t^{-s} \omega^{n}(t, f)_{p}\right]^{q} \frac{d t}{t}\right)^{1 / q}<\infty .
$$

These classes are equipped with a (quasi-)norm by taking

$$
\|f\|_{B_{p, q}^{s}(\mathcal{O})}:=\|f\|_{L_{p}(\mathcal{O})}+|f|_{B_{p, q}^{s}(\mathcal{O})} .
$$

Remark 2. For a more general definition of Besov spaces, including the cases where $p, q=\infty$ and $s<0$ see, e.g. TrIEBEL [45].

We want to describe $B_{p, q}^{s}\left(\mathbb{R}^{d}\right)$ by means of wavelet expansions. To this end let $\varphi$ be a scaling function of tensor product type on $\mathbb{R}^{d}$ and let $\psi_{i}, i=1, \ldots, 2^{d}-1$, be corresponding multivariate mother wavelets, such that, for a given $r \in \mathbb{N}$ and some $N>0$, the following locality, smoothness and vanishing moment conditions hold. For all $i=1, \ldots, 2^{d}-1$,

$$
\begin{aligned}
& \operatorname{supp} \varphi, \operatorname{supp} \psi_{i} \subset[-N, N]^{d}, \\
& \varphi, \psi_{i} \in C^{r}\left(\mathbb{R}^{d}\right), \\
& \int x^{\alpha} \psi_{i}(x) d x=0 \quad \text { for all } \alpha \in \mathbb{N}_{0}^{d} \text { with }|\alpha| \leq r .
\end{aligned}
$$

We assume that

$$
\left\{\varphi_{k}, \psi_{i, j, k}:(i, j, k) \in\left\{1, \cdots, 2^{d}-1\right\} \times \mathbb{N}_{0} \times \mathbb{Z}^{d}\right\}
$$

is a Riesz basis of $L_{2}\left(\mathbb{R}^{d}\right)$, where we use the standard abbreviations for dyadic shifts and dilations of the scaling function and the corresponding wavelets

$$
\begin{aligned}
\varphi_{k}(x) & :=\varphi(x-k), x \in \mathbb{R}^{d}, & & \text { for } k \in \mathbb{Z}^{d}, \text { and } \\
\psi_{i, j, k}(x) & :=2^{j d / 2} \psi_{i}\left(2^{j} x-k\right), x \in \mathbb{R}^{d}, & & \text { for }(i, j, k) \in\left\{1, \cdots, 2^{d}-1\right\} \times \mathbb{N}_{0} \times \mathbb{Z}^{d} .
\end{aligned}
$$

Further, we assume that there exists a dual Riesz basis satisfying the same requirements. More precisely, there exist functions $\widetilde{\varphi}$ and $\widetilde{\psi}_{i}, i=1, \ldots, 2^{d}-1$, such that conditions (4), (5) and (6) hold if $\varphi$ and $\psi$ are replaced by $\widetilde{\varphi}$ and $\widetilde{\psi}_{i}$, and such that the biorthogonality relations

$$
\left\langle\widetilde{\varphi}_{k}, \psi_{i, j, k}\right\rangle=\left\langle\widetilde{\psi}_{i, j, k}, \varphi_{k}\right\rangle=0, \quad\left\langle\widetilde{\varphi}_{k}, \varphi_{\ell}\right\rangle=\delta_{k, \ell}, \quad\left\langle\widetilde{\psi}_{i, j, k}, \psi_{u, v, \ell}\right\rangle=\delta_{i, u} \delta_{j, v} \delta_{k, \ell},
$$

are fulfilled. Here we use analoguous abbreviations to (7) and (8) for the dyadic shifts and dilations of $\widetilde{\varphi}$ and $\widetilde{\psi}_{i}$, and $\delta_{k, l}$ denotes the Kronecker symbol. We refer to CoHen [8, Chapter 2] for the construction of biorthogonal wavelet bases, see also DAUBECHIES [19] and Cohen, Daubechies, Feauveau [11]. To keep notation simple, we will write

$$
\psi_{i, j, k, p}:=2^{j d(1 / p-1 / 2)} \psi_{i, j, k} \quad \text { and } \quad \widetilde{\psi}_{i, j, k, p^{\prime}}:=2^{j d\left(1 / p^{\prime}-1 / 2\right)} \widetilde{\psi}_{i, j, k},
$$


for the $L_{p}$-normalized wavelets and the correspondingly modified duals, with $p^{\prime}:=p /(p-1)$ if $p \in(0, \infty), p \neq 1$, and $p^{\prime}:=\infty, 1 / p^{\prime}:=0$ if $p=1$.

The following theorem shows how Besov spaces can be described by decay properties of the wavelet coefficients, if the parameters fulfil certain conditions.

Theorem 3. Let $p, q \in(0, \infty)$ and $s>\max \{0, d(1 / p-1)\}$. Choose $r \in \mathbb{N}$ such that $r>s$ and construct a biorthogonal wavelet Riesz basis as described above. Then a locally integrable function $f: \mathbb{R}^{d} \rightarrow \mathbb{R}$ is in the Besov space $B_{p, q}^{s}\left(\mathbb{R}^{d}\right)$ if, and only if,

$$
f=\sum_{k \in \mathbb{Z}^{\mathrm{d}}}\left\langle f, \widetilde{\varphi}_{k}\right\rangle \varphi_{k}+\sum_{i=1}^{2^{d}-1} \sum_{j \in \mathbb{N}_{0}} \sum_{k \in \mathbb{Z}^{\mathrm{d}}}\left\langle f, \widetilde{\psi}_{i, j, k, p^{\prime}}\right\rangle \psi_{i, j, k, p}
$$

(convergence in $\mathcal{D}^{\prime}\left(\mathbb{R}^{d}\right)$ ) with

$$
\left(\sum_{k \in \mathbb{Z}^{\mathrm{d}}}\left|\left\langle f, \widetilde{\varphi}_{k}\right\rangle\right|^{p}\right)^{1 / p}+\left(\sum_{i=1}^{2^{d}-1} \sum_{j \in \mathbb{N}_{0}} 2^{j s q}\left(\sum_{k \in \mathbb{Z}^{\mathrm{d}}}\left|\left\langle f, \widetilde{\psi}_{i, j, k, p^{\prime}}\right\rangle\right|^{p}\right)^{q / p}\right)^{1 / q}<\infty
$$

and (10) is an equivalent (quasi-) norm for $B_{p, q}^{s}\left(\mathbb{R}^{d}\right)$.

Remark 4. A proof of this theorem for the case $p \geq 1$ can be found in MEYER [36, $\S 10$ of Chapter 6]. For the general case see for example Kyriazis [34] or CoHEn [8, Theorem 3.7.7]. Of course, if (10) holds then the infinite sum in (9) converges also in $B_{p, q}^{s}\left(\mathbb{R}^{d}\right)$. If $s>\max \{0, d(1 / p-1)\}$ we have the embedding $B_{p, q}^{s}\left(\mathbb{R}^{d}\right) \subset L_{u}\left(\mathbb{R}^{d}\right)$ for some $u>1$, see, e.g. Cohen [8, Corollary 3.7.1].

Let us now fix a value $p \in(1, \infty)$ and consider the scale of Besov spaces $B_{\tau, \tau}^{s}\left(\mathbb{R}^{d}\right)$, $1 / \tau=s / d+1 / p, s>0$. A simple computation gives the following result.

Corollary 5. Let $p \in(1, \infty), s>0$ and $\tau \in \mathbb{R}$ such that $1 / \tau=s / d+1 / p$. Choose $r \in \mathbb{N}$ such that $r>s$ and construct a biorthogonal wavelet Riesz basis as described above. Then a locally integrable function $f: \mathbb{R}^{d} \rightarrow \mathbb{R}$ is in the Besov space $B_{\tau, \tau}^{s}\left(\mathbb{R}^{d}\right)$ if, and only if,

$$
f=\sum_{k \in \mathbb{Z}^{\mathrm{d}}}\left\langle f, \widetilde{\varphi}_{k}\right\rangle \varphi_{k}+\sum_{i=1}^{2^{d}-1} \sum_{j \in \mathbb{N}_{0}} \sum_{k \in \mathbb{Z}^{\mathrm{d}}}\left\langle f, \widetilde{\psi}_{i, j, k, p^{\prime}}\right\rangle \psi_{i, j, k, p}
$$

(convergence in $\mathcal{D}^{\prime}\left(\mathbb{R}^{d}\right)$ ) with

$$
\left(\sum_{k \in \mathbb{Z}^{\mathrm{d}}}\left|\left\langle f, \widetilde{\varphi}_{k}\right\rangle\right|^{\tau}\right)^{1 / \tau}+\left(\sum_{i=1}^{2^{d}-1} \sum_{j \in \mathbb{N}_{0}} \sum_{k \in \mathbb{Z}^{\mathrm{d}}}\left|\left\langle f, \widetilde{\psi}_{i, j, k, p^{\prime}}\right\rangle\right|^{\tau}\right)^{1 / \tau}<\infty,
$$

and (12) is an equivalent (quasi-) norm for $B_{\tau, \tau}^{s}\left(\mathbb{R}^{d}\right)$. 


\subsection{SPDEs on Lipschitz domains and weighted Sobolev spaces}

From now on, let $\mathcal{O} \subset \mathbb{R}^{d}$ be a bounded Lipschitz domain and $d \geq 2$.

We have already mentioned corner singularities as typical examples where the regularity of a function on $\mathcal{O} \subset \mathbb{R}^{d}$ in the Besov scale $B_{\tau, \tau}^{\alpha}(\mathcal{O}), 1 / \tau=\alpha / d+1 / p, \alpha>0$, can exceed the regularity in the Sobolev scale $W_{p}^{s}(\mathcal{O}), s>0$. This reflects the sparsity of the large wavelet coefficients of such a function (given a wavelet basis on the domain $\mathcal{O}$ ). A general way to deal with smoothness regardless of certain singularities at the boundary is to use weighted Sobolev spaces, where the weight function is a power of the distance to the boundary. The $L_{p}$-theory of SPDEs on Lipschitz domains by KIM [30] is based on spaces of this type, namely the weighted Sobolev spaces $H_{p, \theta}^{\gamma}(\mathcal{O}), p \in(1, \infty), \theta, \gamma \in \mathbb{R}$, introduced in Lототsкy [35]. They are defined in terms of the Bessel-potential spaces

$$
H_{p}^{\gamma}\left(\mathbb{R}^{d}\right)=\left\{u \in \mathcal{S}^{\prime}\left(\mathbb{R}^{d}\right):\|u\|_{H_{p}^{\gamma}\left(\mathbb{R}^{d}\right)}=\left\|(1-\Delta)^{\gamma / 2} u\right\|_{L_{p}\left(\mathbb{R}^{d}\right)}<\infty\right\} .
$$

Here, $\mathcal{S}^{\prime}\left(\mathbb{R}^{d}\right) \subset \mathcal{D}^{\prime}\left(\mathbb{R}^{d}\right)$ is the space of (real valued) tempered distributions and $(1-\Delta)^{\gamma / 2}$ : $\mathcal{S}^{\prime}\left(\mathbb{R}^{d}\right) \rightarrow \mathcal{S}^{\prime}\left(\mathbb{R}^{d}\right)$ is the pseudo-differential operator with symbol $\mathbb{R}^{d} \ni \xi \mapsto\left(1+|\xi|^{2}\right)^{\gamma / 2}$, i.e. $(1-\Delta)^{\gamma / 2} u=\mathcal{F}^{-1}\left(\left(1+|\xi|^{2}\right)^{\gamma / 2} \mathcal{F} u\right)$, where $\mathcal{F}$ denotes the Fourier transform on the (complex valued) tempered distributions.

For $x \in \mathcal{O}$ we write $\rho(x):=\operatorname{dist}(x, \partial \mathcal{O})$ for the distance between $x$ and the boundary of the domain $\mathcal{O}$. Fix $c>1, k_{0}>0$ and for $n \in \mathbb{Z}$ consider the subsets $\mathcal{O}_{n}$ of $\mathcal{O}$ given by

$$
\mathcal{O}_{n}:=\left\{x \in \mathcal{O}: c^{-n-k_{0}}<\rho(x)<c^{-n+k_{0}}\right\} .
$$

Let $\zeta_{n}, n \in \mathbb{Z}$, be non-negative functions satisfying $\zeta_{n} \in C_{0}^{\infty}\left(\mathcal{O}_{n}\right), \quad \sum_{n \in \mathbb{Z}} \zeta_{n}(x)=1$ and $\left|D^{m} \zeta_{n}(x)\right| \leq C \cdot c^{m n}$ for all $n \in \mathbb{Z}, m \in \mathbb{N}_{0}, x \in \mathcal{O}$, and a constant $C>0$ that does not depend on $n, m$ and $x$. The functions $\zeta_{n}$ can be constructed by mollifying the indicator functions of the sets $\mathcal{O}_{n}$, see, e.g. Hörmander [27, Section 1.4]. If $\mathcal{O}_{n}$ is empty we set $\zeta_{n} \equiv 0$. For $u \in \mathcal{D}^{\prime}(\mathcal{O}) \zeta_{n} u$ is a distribution on $\mathcal{O}$ with compact support which can be extended by zero to $\mathbb{R}^{d}$. This extension is a tempered distribution, i.e. $\zeta_{n} u \in \mathcal{S}^{\prime}\left(\mathbb{R}^{d}\right)$.

Definition 6. Let $\zeta_{n}, n \in \mathbb{Z}$, be as above and $p \in(1, \infty), \theta, \gamma \in \mathbb{R}$. Then

$$
H_{p, \theta}^{\gamma}(\mathcal{O}):=\left\{u \in \mathcal{D}^{\prime}(\mathcal{O}):\|u\|_{H_{p, \theta}^{\gamma}(\mathcal{O})}^{p}:=\sum_{n \in \mathbb{Z}} c^{n \theta}\left\|\zeta_{-n}\left(c^{n} \cdot\right) u\left(c^{n} \cdot\right)\right\|_{H_{p}^{\gamma}\left(\mathbb{R}^{d}\right)}^{p}<\infty\right\} .
$$

According to Lototsky [35] this definition is independent of the specific choice of $c, k_{0}$ and $\zeta_{n}, n \in \mathbb{N}_{0}$, in the sense that one gets equivalent norms. If $\gamma=m \in \mathbb{N}_{0}$ then the spaces can be characterized as

$$
\begin{aligned}
& H_{p, \theta}^{0}(\mathcal{O})=L_{p, \theta}(\mathcal{O}):=L_{p}\left(\mathcal{O}, \rho(x)^{\theta-d} d x\right), \\
& H_{p, \theta}^{m}(\mathcal{O})=\left\{u: \rho^{|\alpha|} D^{\alpha} u \in L_{p, \theta}(\mathcal{O}) \text { for all } \alpha \in \mathbb{N}_{0}^{d} \text { with }|\alpha| \leq m\right\},
\end{aligned}
$$

and one has the norm equivalence

$$
C^{-1}\|u\|_{H_{p, \theta}^{m}(\mathcal{O})}^{p} \leq \sum_{\alpha \in \mathbb{N}_{0}^{d},|\alpha| \leq m} \int_{\mathcal{O}}\left|\rho(x)^{|\alpha|} D^{\alpha} u(x)\right|^{p} \rho(x)^{\theta-d} d x \leq C\|u\|_{H_{p, \theta}^{m}(\mathcal{O})}^{p} .
$$


Analogous notations are used for $\ell_{2}=\ell_{2}(\mathbb{N})$-valued functions $g=\left(g^{\kappa}\right)_{\kappa \in \mathbb{N}}$. For $p \in$ $(1, \infty), \theta, \gamma \in \mathbb{R}$ and $\zeta_{n}, n \in \mathbb{Z}$, as above

$$
\begin{aligned}
& H_{p}^{\gamma}\left(\mathbb{R}^{d} ; \ell_{2}\right):=\left\{g \in\left(\mathcal{S}^{\prime}\left(\mathbb{R}^{d}\right)\right)^{\mathbb{N}}:(1-\Delta)^{\gamma / 2} g^{\kappa} \in L_{p}\left(\mathbb{R}^{d}\right) \text { for all } k \in \mathbb{N}\right. \text { and } \\
& \left.\|g\|_{H_{p}^{\gamma}\left(\mathbb{R}^{d} ; \ell_{2}\right)}:=\left\|\left|\left((1-\Delta)^{\gamma / 2} g^{\kappa}\right)_{\kappa \in \mathbb{N}}\right|_{\ell_{2}}\right\|_{L_{p}\left(\mathbb{R}^{d}\right)}<\infty\right\}, \\
& H_{p, \theta}^{\gamma}\left(\mathcal{O} ; \ell_{2}\right):=\left\{g \in\left(\mathcal{D}^{\prime}(\mathcal{O})\right)^{\mathbb{N}}:\|g\|_{H_{p, \theta}^{\gamma}\left(\mathcal{O} ; \ell_{2}\right)}^{p}:=\sum_{n \in \mathbb{Z}} c^{n \theta}\left\|\zeta_{-n}\left(c^{n} \cdot\right) g\left(c^{n} \cdot\right)\right\|_{H_{p}^{\gamma}\left(\mathbb{R}^{d} ; \ell_{2}\right)}^{p}<\infty\right\} .
\end{aligned}
$$

Remark 7. (a) One can consider the spaces $H_{p, \theta}^{\gamma}(\mathcal{O})$ as generalizations of the classical Sobolev spaces on $\mathcal{O}$ with zero boundary conditions. For $\gamma=m \in \mathbb{N}_{0}$ we have the identity

$$
H_{p, d-m p}^{m}(\mathcal{O})=\stackrel{\circ}{W}_{p}^{m}(\mathcal{O}),
$$

and the norms in both spaces are equivalent, see Theorem 9.7. in KufNer [33]. Here $\stackrel{\circ}{W}_{p}^{m}(\mathcal{O})$ is the closure of $C_{0}^{\infty}(\mathcal{O})$ in the classical Sobolev space $W_{p}^{m}(\mathcal{O})$.

(b) Note that, in contrast to the spaces $W_{p}^{s}(\mathcal{O})=B_{p, p}^{s}(\mathcal{O}), s \in(m, m+1), m \in$ $\mathbb{N}_{0}$, which can be regarded as real interpolation spaces of the classical Sobolev spaces $W_{p}^{m}(\mathcal{O}), m \in \mathbb{N}_{0}$ (see, e.g. TrIEBEL [45, Section 1.11.8] and Dispa[23]), the spaces $H_{p, \theta}^{\gamma}(\mathcal{O}), \quad \gamma \in(m, m+1), m \in \mathbb{N}_{0}$, are complex interpolants of the respective integer smoothness spaces (cf. Lototsky [35, Proposition 2.4]).

We can now define spaces of stochastic processes and random functions in terms of the weighted Sobolev spaces introduced above.

Definition 8. For $\gamma, \theta \in \mathbb{R}$ and $p \in(1, \infty)$ we set

$$
\begin{aligned}
\mathbb{H}_{p, \theta}^{\gamma}(\mathcal{O}, T) & :=L_{p}\left(\Omega_{T}, \mathcal{P}, \mathbb{P} \otimes \lambda ; H_{p, \theta}^{\gamma}(\mathcal{O})\right), \\
\mathbb{H}_{p, \theta}^{\gamma}\left(\mathcal{O}, T ; \ell_{2}\right) & :=L_{p}\left(\Omega_{T}, \mathcal{P}, \mathbb{P} \otimes \lambda ; H_{p, \theta}^{\gamma}\left(\mathcal{O} ; \ell_{2}\right)\right), \\
U_{p, \theta}^{\gamma}(\mathcal{O}) & :=L_{p}\left(\Omega, \mathcal{F}_{0}, \mathbb{P} ; H_{p, \theta+2-p}^{\gamma-2 / p}(\mathcal{O})\right),
\end{aligned}
$$

and for $p \in[2, \infty)$,

$$
\begin{array}{r}
\mathfrak{H}_{p, \theta}^{\gamma}(\mathcal{O}, T):=\left\{u \in \mathbb{H}_{p, \theta-p}^{\gamma}(\mathcal{O}, T): u(0, \cdot) \in U_{p, \theta}^{\gamma}(\mathcal{O}) \text { and } d u=f d t+\sum_{\kappa=1}^{\infty} g^{\kappa} d w_{t}^{\kappa}\right. \\
\text { for some } \left.f \in \mathbb{H}_{p, \theta+p}^{\gamma-2}(\mathcal{O}, T), g \in \mathbb{H}_{p, \theta}^{\gamma-1}\left(\mathcal{O}, T ; \ell_{2}\right)\right\}
\end{array}
$$

equipped with the norm

$$
\|u\|_{\mathfrak{H}_{p, \theta}^{\gamma}(\mathcal{O}, T)}:=\|u\|_{\mathbb{H}_{p, \theta-p}^{\gamma}(\mathcal{O}, T)}+\|f\|_{\mathbb{H}_{p, \theta+p}^{\gamma-2}(\mathcal{O}, T)}+\|g\|_{\mathbb{H}_{p, \theta}^{\gamma-1}\left(\mathcal{O}, T ; \ell_{2}\right)}+\|u(0, \cdot)\|_{U_{p, \theta}^{\gamma}(\mathcal{O})} \cdot
$$

The equality $d u=f d t+\sum_{\kappa=1}^{\infty} g^{\kappa} d w_{t}^{\kappa}$ above is a shorthand for

$$
\langle u(t, \cdot), \varphi\rangle=\langle u(0, \cdot), \varphi\rangle+\int_{0}^{t}\langle f(s, \cdot), \varphi\rangle d s+\sum_{\kappa=1}^{\infty} \int_{0}^{t}\left\langle g^{\kappa}(s, \cdot), \varphi\right\rangle d w_{s}^{\kappa}
$$

for all $\varphi \in C_{0}^{\infty}(\mathcal{O}), t \in[0, T]$. 
Remark 9. (a) If $p \in[2, \infty)$, then the sum of stochastic integrals in (14) converges in the space $\mathcal{M}_{T}^{2, c}\left(\mathbb{R},\left(\mathcal{F}_{t}\right)\right)$ of continuous, square integrable, $\mathbb{R}$-valued martingales w.r.t $\left(\mathcal{F}_{t}\right)_{t \in[0, T]}$. For the convenience of the readers we include a proof in Appendix A.

(b) Using the arguments of KRYLOV in [31, Remark 3.3], we get the uniqueness (up to indistinguishability) of the pair $(f, g) \in \mathbb{H}_{p, \theta+p}^{\gamma-2}(\mathcal{O}, T) \times \mathbb{H}_{p, \theta}^{\gamma-1}\left(\mathcal{O}, T ; \ell_{2}\right)$ which fulfils (14). Consequently, the norm in $\mathfrak{H}_{p, \theta}^{\gamma}(\mathcal{O}, T)$ is well defined.

Definition 10. We call a predictable $\mathcal{D}^{\prime}(\mathcal{O})$-valued stochastic process $u=(u(t, \cdot))_{t \in[0, T]}$ a solution of equation (11) if it is a solution of equation (14) where $f$ is replaced by $\sum_{\mu, \nu=1}^{d} a^{\mu \nu} u_{x_{\mu} x_{\nu}}$ and $u(0, \cdot)=u_{0}$.

The next result is taken from KIM [30].

Theorem 11. Let $p \in[2, \infty)$ and $\gamma \in \mathbb{R}$. There exists a constant $\kappa_{0} \in(0,1)$, depending only on $d, p,\left(a^{\mu \nu}\right)_{1 \leq \mu, \nu \leq d}$ and $\mathcal{O}$, such that for any $\theta \in\left(d-\kappa_{0}, d-2+p+\kappa_{0}\right)$, $g \in \mathbb{H}_{p, \theta}^{\gamma-1}\left(\mathcal{O}, T ; \ell_{2}\right)$ and $u_{0} \in U_{p, \theta}^{\gamma}(\mathcal{O})$ the equation (1) has a unique solution in the class $\mathfrak{H}_{p, \theta}^{\gamma}(\mathcal{O}, T)$.

For this solution

$$
\|u\|_{\mathfrak{H}_{p, \theta}^{\gamma}(\mathcal{O}, T)}^{p} \leq C\left(\|g\|_{\mathbb{H}_{p, \theta}^{\gamma-1}\left(\mathcal{O}, T ; \ell_{2}\right)}^{p}+\left\|u_{0}\right\|_{U_{p, \theta}^{\gamma}(\mathcal{O})}^{p}\right)
$$

where the constant $C$ depends only on $d, p, \gamma, \theta,\left(a^{\mu \nu}\right)_{1 \leq \mu, \nu \leq d}, T$ and $\mathcal{O}$.

We will need the following straightforward consequence of this Theorem 11. Recall that if $m \in \mathbb{N}$ and $f \in \mathcal{D}^{\prime}(\mathcal{O})$ is sufficient regular, then $\left|D^{m} f\right|_{\ell_{p}}$ stands for $\left(\sum_{|\alpha|=m}\left|D^{\alpha} f\right|^{p}\right)^{1 / p}$, the (pointwise) $\ell_{p}$-norm of the vector of the $m$-th order derivatives of $f$.

Corollary 12. In the situation of Theorem 11 with $\gamma=m \in \mathbb{N}$, the following inequality holds for every $\tau \in[0, p]$.

$$
\int_{\Omega} \int_{0}^{T}\left\|\rho^{m-\delta}\left|D^{m} u(\omega, t, \cdot)\right|_{\ell_{p}}\right\|_{L_{p}(\mathcal{O})}^{\tau} d t \mathbb{P}(d \omega) \leq C\left(\|g\|_{\mathbb{H}_{p, \theta}^{m-1}\left(\mathcal{O}, T ; \ell_{2}\right)}+\left\|u_{0}\right\|_{U_{p, \theta}^{m}(\mathcal{O})}\right)^{\tau},
$$

where $\delta=1+\frac{d-\theta}{p}$.

Proof. Theorem 11 implies, in particular, that

$$
\|u\|_{\mathbb{H}_{p, \theta-p}^{m}(\mathcal{O}, T)} \leq C\left(\|g\|_{\mathbb{H}_{p, \theta}^{m-1}(\mathcal{O}, T)}+\left\|u_{0}\right\|_{U_{p, \theta}^{m}(\mathcal{O})}\right)
$$

and we have

$$
\begin{aligned}
\|u\|_{\mathbb{H}_{p, \theta-p}^{m}(\mathcal{O}, T)}^{p} & =\int_{\Omega} \int_{0}^{T}\|u(\omega, t, \cdot)\|_{H_{p, \theta-p}^{m}(\mathcal{O}, T)}^{p} d t \mathbb{P}(d \omega) \\
& \geq C \int_{\Omega} \int_{0}^{T} \sum_{k=0}^{m}\left\|\rho^{k+(\theta-p-d) / p}\left|D^{k} u(\omega, t, \cdot)\right|_{\ell_{p}}\right\|_{L_{p}(\mathcal{O})}^{p} d t \mathbb{P}(d \omega)
\end{aligned}
$$




$$
\geq C \int_{\Omega} \int_{0}^{T}\left\|\rho^{m-\delta}\left|D^{m} u(\omega, t, \cdot)\right|_{\ell_{p}}\right\|_{L_{p}(\mathcal{O})}^{p} d t \mathbb{P}(d \omega)
$$

with $\delta=1+\frac{d-\theta}{p} \in\left(\left(2-\kappa_{0}\right) / p,\left(p+\kappa_{0}\right) / p\right)$. Now let $\tau \in[0, p]$. Jensen's inequality for concave functions, see, e.g. Schilling [42, Theorem 12.14], yields

$$
\begin{aligned}
\int_{\Omega} \int_{0}^{T}\left\|\rho^{m-\delta}\left|D^{m} u(\omega, t, \cdot)\right|_{\ell_{p}}\right\|_{L_{p}(\mathcal{O})}^{\tau} d t \mathbb{P}(d \omega) & \leq C(T)\left(\|g\|_{\mathbb{H}_{p, \theta}^{m-1}(\mathcal{O}, T)}^{p}+\left\|u_{0}\right\|_{U_{p, \theta}^{m}(\mathcal{O})}^{p}\right)^{\tau / p} \\
& \leq C\left(\|g\|_{\mathbb{H}_{p, \theta}^{m-1}(\mathcal{O}, T)}+\left\|u_{0}\right\|_{U_{p, \theta}^{m}(\mathcal{O})}\right)^{\tau} .
\end{aligned}
$$

In the last step we have used the fact that all norms on $\mathbb{R}^{2}$ are equivalent.

Remark 13. Consider the Hilbert space case $p=2$ and assume $g \in \mathbb{H}_{2, \theta}^{\gamma}\left(\mathcal{O}, T ; \ell_{2}\right)$. The expression $\sum_{\kappa=1}^{\infty} \int_{0}^{t} g^{\kappa}(s, \cdot) d w_{s}^{\kappa}$ can be considered as an $\mathbb{H}_{2, \theta}^{\gamma}(\mathcal{O})$-valued stochastic integral $\int_{0}^{t} G(s) d W_{s}$ with respect to a cylindrical Wiener process $\left(W_{t}\right)_{t \in[0, T]}$ on $\ell_{2}$ whose coordinate processes are $\left(w_{t}^{\kappa}\right)_{t \in[0, T]}, \kappa \in \mathbb{N}$. (See, e.g. DA PrAto, ZABCZYK [18] or PeszAT, ZABCZYK [40] for stochastic integration w.r.t. cylindrical processes.) Here $(G(t))_{t \in[0, T]}$ is a stochastic process in the space of Hilbert-Schmidt operators $L_{(\mathrm{HS})}\left(\ell_{2}, H_{2, \theta}^{\gamma}(\mathcal{O})\right)$ defined by

$$
G(\omega, t): \ell_{2} \rightarrow H_{2, \theta}^{\gamma}(\mathcal{O}),\left(x^{\kappa}\right)_{\kappa \in \mathbb{N}} \mapsto \sum_{\kappa \in \mathbb{N}} g^{\kappa}(\omega, t, \cdot) x^{\kappa}, \quad(\omega, t) \in \Omega_{T},
$$

and it is an element of the space $L_{2}\left(\Omega_{T} ; L_{(\mathrm{HS})}\left(\ell_{2}, H_{2, \theta}^{\gamma}(\mathcal{O})\right)\right)$. Indeed, for fixed $(\omega, t) \in \Omega_{T}$ we have

$$
\begin{aligned}
\|G(\omega, t)\|_{L_{(\mathrm{HS})}\left(\ell_{2}, H_{2, \theta}^{\gamma}(\mathcal{O})\right)}^{2} & =\sum_{\kappa \in \mathbb{N}}\left\|g^{\kappa}(\omega, t, \cdot)\right\|_{H_{2, \theta}^{\gamma}(\mathcal{O})}^{2} \\
& =\sum_{\kappa \in \mathbb{N}} \sum_{n \in \mathbb{Z}} c^{n \theta}\left\|\zeta_{-n}\left(c^{n} \cdot\right) g^{\kappa}\left(\omega, t, c^{n} \cdot\right)\right\|_{H_{2}^{\gamma}\left(\mathbb{R}^{d}\right)}^{2} \\
& =\sum_{n \in \mathbb{Z}} c^{n \theta}\left\|\zeta_{-n}\left(c^{n} \cdot\right) g\left(\omega, t, c^{n} \cdot\right)\right\|_{H_{2}^{\gamma}\left(\mathbb{R}^{d} ; \ell_{2}\right)}^{2}
\end{aligned}
$$

by Tonelli's theorem, so that

$$
\|G\|_{L_{2}\left(\Omega_{T} ; L_{(\mathrm{HS})}\left(\ell_{2} ; H_{2, \theta}^{\gamma}(\mathcal{O})\right)\right)}=\|g\|_{\mathbb{H}_{2, \theta}^{\gamma}\left(\mathcal{O}, T ; \ell_{2}\right)} .
$$

As a consequence, equation (11) can be rewritten in the form

$$
d u=\sum_{\mu, \nu=1}^{d} a^{\mu \nu} u_{x_{\mu} x_{\nu}} d t+d M_{t}, \quad u(0, \cdot)=u_{0},
$$

where $\left(M_{t}\right)_{t \in[0, T]} \in \mathcal{M}_{T}^{2, c}\left(H_{2, \theta}^{\gamma}(\mathcal{O}),\left(\mathcal{F}_{t}\right)\right)$ is the $H_{2, \theta}^{\gamma}(\mathcal{O})$-valued, square-integrable martingale given by

$$
M_{t}:=\int_{0}^{t} G(s) d W_{s}, \quad t \in[0, T] .
$$


Remark 14. In Examples 17, 18 and 19 below the solution $u$ of equation (11) in $\mathfrak{H}_{2, \theta}^{\gamma}(\mathcal{O}, T)$ as given by Theorem 11 coincides with the weak solution of equation (16) with zero Dirichlet boundary condition in the sense of DA PRATO, ZABCZYK [18].

In the examples we consider equation (16) driven by certain Wiener processes $\left(M_{t}\right)_{t \in[0, T]}$ in $L_{2}(\mathcal{O})$ with $u_{0} \in U_{2,2}^{2}(\mathcal{O}), d=2$ and the solution $u$ is in the class $\mathfrak{H}_{2,2}^{2}(\mathcal{O}, T) \subset$ $\mathrm{H}_{2,0}^{2}(\mathcal{O}, T)$. (Strictly speaking, in Example $19\left(M_{t}\right)_{t \in[0, T]}$ is not a Wiener process, but it is one conditioned on the family of random variables $Y_{\lambda}, \lambda \in \nabla$.) Thus, by Remark 7 (a) we know that $u$ is an element of $L_{2}\left(\Omega_{T} ; \stackrel{\circ}{W}_{2}^{1}(\mathcal{O})\right)$. Let us now introduce the operator

$$
(A, D(A)):=\left(\sum_{\mu, \nu=1}^{d} a^{\mu \nu} \frac{\partial^{2}}{\partial x_{\mu} \partial x_{\nu}},\left\{u \in \stackrel{\circ}{W}_{2}^{1}(\mathcal{O}): \sum_{\mu, \nu=1}^{d} a^{\mu \nu} u_{x_{\mu} x_{\nu}} \in L_{2}(\mathcal{O})\right\}\right)
$$

and consider the equation

$$
d u(t, \cdot)=A u(t, \cdot) d t+d M_{t}, \quad u(0, \cdot)=u_{0} \in L_{2}(\mathcal{O}), t \in[0, T] .
$$

A weak solution of equation (17) in the sense of DA PRATO, ZABCZYK [18] is an $L_{2}(\mathcal{O})$ valued predictable process $u=(u(t, \cdot))_{t \in[0, T]}$ with $\mathbb{P}$-almost surely Bochner integrable trajectories $t \mapsto u(\omega, t, \cdot)$ satisfying

$$
\langle u(t, \cdot), \zeta\rangle_{L_{2}(\mathcal{O})}=\left\langle u_{0}, \zeta\right\rangle_{L_{2}(\mathcal{O})}+\int_{0}^{t}\left\langle u(s, \cdot), A^{*} \zeta\right\rangle_{L_{2}(\mathcal{O})} d s+\left\langle M_{t}, \zeta\right\rangle_{L_{2}(\mathcal{O})}
$$

for all $t \in[0, T]$ and $\zeta \in D\left(A^{*}\right)$. It is given by the variation of constants formula

$$
u(t, \cdot)=e^{t A} u_{0}+\int_{0}^{t} e^{(t-s) A} d M_{s}, \quad t \in[0, T],
$$

where $\left(e^{t A}\right)_{t \geq 0}$ is the contraction semigroup on $L_{2}(\mathcal{O})$ generated by $A$.

It is clear that the solution $u \in \mathfrak{H}_{2,2}^{2}(\mathcal{O}, T)$ given by Theorem 11 satisfies

$$
\langle u(t, \cdot), \varphi\rangle_{L_{2}(\mathcal{O})}=\left\langle u_{0}, \varphi\right\rangle_{L_{2}(\mathcal{O})}+\int_{0}^{t}\left\langle A^{1 / 2} u(s, \cdot), A^{1 / 2} \varphi\right\rangle_{L_{2}(\mathcal{O})} d s+\left\langle M_{t}, \varphi\right\rangle_{L_{2}(\mathcal{O})}
$$

for all $t \in[0, T]$ and $\varphi \in C_{0}^{\infty}(\mathcal{O})$. Note that the operator $A$ is self-adjoint because the coefficients $a^{\mu \nu}, 1 \leq \mu, \nu \leq d$, are constants. Since every $\zeta \in D\left(A^{*}\right)=D(A) \subset \stackrel{\circ}{2}_{2}^{1}(\mathcal{O})$ is the limit in $W_{2}^{1}(\mathcal{O})$ of a sequence of test functions $\left(\varphi_{k}\right)_{k \in \mathbb{N}} \subset C_{0}^{\infty}(\mathcal{O})$, one can go to the limit $k \rightarrow \infty$ for $\varphi=\varphi_{k}$ in (19) to obtain equation (18). 


\section{Besov regularity for SPDEs}

In this section we state and prove our main result. We give some concrete examples to illustrate its applicability. The result is formulated in terms of the $L_{\tau}$-spaces

$$
L_{\tau}\left(\Omega_{T} ; B_{\tau, \tau}^{s}(\mathcal{O})\right)=L_{\tau}\left(\Omega_{T}, \mathcal{P}, \mathbb{P} \otimes \lambda ; B_{\tau, \tau}^{s}(\mathcal{O})\right), \quad \tau \in(0, \infty), s \in(0, \infty),
$$

and the spaces introduced in the last section.

Theorem 15. Let $p \in[2, \infty)$ and $g \in \mathbb{H}_{p, \theta}^{\gamma-1}\left(\mathcal{O}, T ; \ell_{2}\right), u_{0} \in U_{p, \theta}^{\gamma}(\mathcal{O})$ for some $\gamma \in \mathbb{N}$ and $\theta \in\left(d-\kappa_{0}, d-2+p+\kappa_{0}\right)$ with $\kappa_{0}=\kappa_{0}\left(d, p,\left(a^{\mu \nu}\right), \mathcal{O}\right) \in(0,1)$ as in Theorem 11. Let $u$ be the unique solution in the class $\mathfrak{H}_{p, \theta}^{\gamma}(\mathcal{O}, T)$ of equation (11) and assume furthermore that

$$
u \in L_{p}\left(\Omega_{T} ; B_{p, p}^{s}(\mathcal{O})\right) \quad \text { for some } \quad s \in\left(0, \gamma \wedge\left(1+\frac{d-\theta}{p}\right)\right] .
$$

Then, we have

$$
u \in L_{\tau}\left(\Omega_{T} ; B_{\tau, \tau}^{\alpha}(\mathcal{O})\right), \quad \frac{1}{\tau}=\frac{\alpha}{d}+\frac{1}{p}, \quad \text { for all } \quad \alpha \in\left(0, \gamma \wedge \frac{s d}{d-1}\right),
$$

and the following estimate holds

$$
\|u\|_{L_{\tau}\left(\Omega_{T} ; B_{\tau, \tau}^{\alpha}(\mathcal{O})\right)} \leq C\left(\|g\|_{\mathbb{H}_{p, \theta}^{\gamma-1}\left(\mathcal{O}, T ; \ell_{2}\right)}+\left\|u_{0}\right\|_{U_{p, \theta}^{\gamma}(\mathcal{O})}+\|u\|_{L_{p}\left(\Omega_{T} ; B_{p, p}^{s}(\mathcal{O})\right)}\right) .
$$

Here the constant $C$ depends only on $d, p, \gamma, \alpha, s, \theta,\left(a^{\mu \nu}\right)_{1 \leq \mu, \nu \leq d}, T$ and $\mathcal{O}$.

Remark 16. Since the constant $\kappa_{0}=\kappa_{0}\left(d, p,\left(a^{\mu \nu}\right), \mathcal{O}\right)$ is greater than zero, we can always choose $\theta=d$. In this case, we know from Theorem 11 that for each $\gamma \in \mathbb{N}$ we have a unique solution $u$ in the class $\mathfrak{H}_{p, d}^{\gamma}(\mathcal{O}, T)$, provided the free term $g$ and the initial condition $u_{0}$ are sufficiently regular. In particular, we get

$$
u \in \mathbb{H}_{p, d-p}^{\gamma}(\mathcal{O}, T)=L_{p}\left(\Omega_{T}, \mathcal{P}, \mathbb{P} \otimes \lambda ; H_{p, d-p}^{\gamma}(\mathcal{O})\right) \subseteq L_{p}\left(\Omega_{T} ; W_{p}^{1}(\mathcal{O})\right) \subseteq L_{p}\left(\Omega_{T} ; B_{p, p}^{1}(\mathcal{O})\right) .
$$

Thus, the additional requirement (20) is fulfilled with $s=1$. Since $\mathcal{O}$ is an arbitrary bounded Lipschitz domain it is in general not clear if $u$ belongs to $L_{p}\left(\Omega_{T} ; W_{p}^{s}(\mathcal{O})\right)$ for all $s<2$, compare Example 17 below.

However, if $\gamma \geq 2$ our result shows that we obtain higher regularity than $s=1$ in the nonlinear approximation scale, namely

$$
u \in L_{\tau}\left(\Omega_{T} ; B_{\tau, \tau}^{\alpha}(\mathcal{O})\right), \quad \frac{1}{\tau}=\frac{\alpha}{d}+\frac{1}{p}, \quad \text { for all } \quad \alpha<\frac{d}{d-1} .
$$


Proof of Theorem 15. We fix $\alpha$ and $\tau$ as stated in the theorem and choose a wavelet Rieszbasis

$$
\left\{\varphi_{k}, \psi_{i, j, k}:(i, j, k) \in\left\{1, \cdots, 2^{d}-1\right\} \times \mathbb{N}_{0} \times \mathbb{Z}^{d}\right\}
$$

of $L_{2}\left(\mathbb{R}^{d}\right)$ which fulfils the assumptions from Section 2.2 with $r>\gamma$. Given $(j, k) \in \mathbb{N}_{0} \times \mathbb{Z}^{\mathrm{d}}$ let

$$
Q_{j, k}:=2^{-j} k+2^{-j}[-N, N]^{d},
$$

such that $\operatorname{supp} \psi_{i, j, k} \subset Q_{j, k}$ for all $i \in\left\{1, \ldots, 2^{d}-1\right\}$ and supp $\varphi_{k} \subset Q_{0, k}$ for all $k \in \mathbb{Z}^{\mathrm{d}}$. Remember that the supports of the corresponding dual basis fulfil the same requirements. For our purpose the set of all indices associated with that wavelets that may have common support with the domain $\mathcal{O}$ will play an important role and we denote them by

$$
\Lambda:=\left\{(i, j, k) \in\left\{1, \ldots, 2^{d}-1\right\} \times \mathbb{N}_{0} \times \mathbb{Z}^{\mathrm{d}} \mid Q_{j, k} \cap \mathcal{O} \neq \emptyset\right\} .
$$

In particular, we will also use the following notation:

$$
\Gamma:=\left\{k \in \mathbb{Z}^{\mathrm{d}}: Q_{0, k} \cap \mathcal{O} \neq \emptyset\right\}
$$

Due to the assumption $u \in L_{p}\left(\Omega_{T} ; B_{p, p}^{s}(\mathcal{O})\right)$ we have $u(\omega, t, \cdot) \in B_{p, p}^{s}(\mathcal{O})$ for $\mathbb{P} \otimes \lambda$ almost every $(\omega, t) \in \Omega_{T}$. As $\mathcal{O}$ is a Lipschitz domain there exists a linear and bounded extension operator $\mathcal{E}: B_{p, p}^{s}(\mathcal{O}) \rightarrow B_{p, p}^{s}\left(\mathbb{R}^{d}\right)$, i.e. there exists a constant $C>0$ such that for $\mathbb{P} \otimes \lambda$-almost every $(\omega, t) \in \Omega_{T}$ :

$$
\left.\mathcal{E} u(\omega, t, \cdot)\right|_{\mathcal{O}}=u(\omega, t, \cdot) \quad \text { and } \quad\|\mathcal{E} u(\omega, t, \cdot)\|_{B_{p, p}^{s}\left(\mathbb{R}^{d}\right)} \leq C\|u(\omega, t, \cdot)\|_{B_{p, p}^{s}(\mathcal{O})},
$$

see, e.g. RYCHKOV [41]. In the sequel we will omit the $\mathcal{E}$ in our notation and write $u$ instead of $\mathcal{E} u$.

Theorem 3 tells us that for almost all $(\omega, t) \in \Omega_{T}$ the following equality holds on the domain $\mathcal{O}$

$$
u(\omega, t, \cdot)=\sum_{k \in \Gamma}\left\langle u(\omega, t, \cdot), \widetilde{\varphi}_{k}\right\rangle \varphi_{k}+\sum_{(i, j, k) \in \Lambda}\left\langle u(\omega, t, \cdot), \widetilde{\psi}_{i, j, k, p^{\prime}}\right\rangle \psi_{i, j, k, p}
$$

where the sums converge unconditionally in $B_{p, p}^{s}\left(\mathbb{R}^{d}\right)$. Furthermore, cf. Corollary 5 , we get for $\mathbb{P} \otimes \lambda$-almost all $(\omega, t) \in \Omega_{T}$

$$
\|u(\omega, t, \cdot)\|_{B_{\tau, \tau}^{\alpha}(\mathcal{O})}^{\tau} \leq C\left(\sum_{k \in \Gamma}\left|\left\langle u(\omega, t, \cdot), \widetilde{\varphi}_{k}\right\rangle\right|^{\tau}+\sum_{(i, j, k) \in \Lambda}\left|\left\langle u(\omega, t, \cdot), \widetilde{\psi}_{i, j, k, p^{\prime}}\right\rangle\right|^{\tau}\right) .
$$

Hence, it is enough to prove that

$$
\int_{\Omega} \int_{0}^{T} \sum_{k \in \Gamma}\left|\left\langle u(\omega, t, \cdot), \widetilde{\varphi}_{k}\right\rangle\right|^{\tau} d t \mathbb{P}(d \omega) \leq C\|u\|_{L_{p}\left(\Omega_{T} ; B_{p, p}^{s}(\mathcal{O})\right)}^{\tau}
$$


and

$$
\begin{aligned}
& \int_{\Omega} \int_{0}^{T} \sum_{(i, j, k) \in \Lambda}\left|\left\langle u(\omega, t, \cdot), \widetilde{\psi}_{i, j, k, p^{\prime}}\right\rangle\right|^{\tau} d t \mathbb{P}(d \omega) \\
& \leq C\left(\|g\|_{\mathbb{H}_{p, \theta}^{\gamma-1}\left(\mathcal{O}, T ; \ell_{2}\right)}+\left\|u_{0}\right\|_{U_{p, \theta}^{\gamma}(\mathcal{O})}+\|u\|_{L_{p}\left(\Omega_{T} ; B_{p, p}^{s}(\mathcal{O})\right)}\right)^{\tau} .
\end{aligned}
$$

We start with (23). The index set $\Gamma$ introduced above is finite because of the boundedness of the domain $\mathcal{O}$, so that we can use Jensen's inequality to get for $\mathbb{P} \otimes \lambda$-almost all $(\omega, t) \in \Omega_{T}$

$$
\sum_{k \in \Gamma}\left|\left\langle u(\omega, t, .), \widetilde{\varphi}_{k}\right\rangle\right|^{\tau} \leq C\left(\left(\sum_{k \in \Gamma}\left|\left\langle u(\omega, t, .), \widetilde{\varphi}_{k}\right\rangle\right|^{p}\right)^{1 / p}\right)^{\tau} \leq C\|u(\omega, t, .)\|_{B_{p, p}^{s}(\mathcal{O})}^{\tau} .
$$

In the last step we used Theorem 3 and the boundedness of the extension operator. Integration with respect to $\mathbb{P} \otimes \lambda$ and another application of Jensen's inequality yield (23).

Now let us focus on the inequality (24). To this end, we introduce the following notation

$$
\begin{aligned}
\rho_{j, k} & :=\operatorname{dist}\left(Q_{j, k}, \partial \mathcal{O}\right)=\inf _{x \in Q_{j, k}} \rho(x), \\
\Lambda_{j} & :=\{(i, l, k) \in \Lambda: l=j\} \\
\Lambda_{j, m} & :=\left\{(i, j, k) \in \Lambda_{j}: m 2^{-j} \leq \rho_{j, k}<(m+1) 2^{-j}\right\}, \\
\Lambda_{j}^{0} & :=\Lambda_{j} \backslash \Lambda_{j, 0}, \\
\Lambda^{0} & :=\bigcup_{j \in \mathbb{N}_{0}} \Lambda_{j}^{0},
\end{aligned}
$$

where $j, m \in \mathbb{N}_{0}$ and $k \in \mathbb{Z}^{\mathrm{d}}$. We split the expression on the left hand side of (24) into

$$
\begin{aligned}
\int_{\Omega} \int_{0}^{T} \sum_{(i, j, k) \in \Lambda^{0}} & \left|\left\langle u(\omega, t, \cdot), \widetilde{\psi}_{i, j, k, p^{\prime}}\right\rangle\right|^{\tau} d t \mathbb{P}(d \omega) \\
& +\int_{\Omega} \int_{0}^{T} \sum_{(i, j, k) \in \Lambda \backslash \Lambda^{0}}\left|\left\langle u(\omega, t, \cdot), \widetilde{\psi}_{i, j, k, p^{\prime}}\right\rangle\right|^{\tau} d t \mathbb{P}(d \omega)=: I+I I
\end{aligned}
$$

and estimate each term separately.

Let us begin with $I$. Fix $(i, j, k) \in \Lambda^{0}$ and $(\omega, t) \in \Omega_{T}$ such that

$$
\left.\left.\int_{\mathcal{O}}\left|\rho(x)^{\gamma-s}\right| D^{\gamma} u(\omega, t, x)\right|_{\ell_{p}}\right|^{p} d x<\infty
$$

By Corollary 12 this holds for $\mathbb{P} \otimes \lambda$-almost all $(\omega, t) \in \Omega_{T}$. By a Whitney-type inequality, also known as the Deny-Lions lemma, see, e.g. DeVore, Sharpley [22, Theorem 3.4], there exists a polynomial $P_{j, k}$ of total degree less than $\gamma$ such that

$$
\left\|u(\omega, t, \cdot)-P_{j, k}\right\|_{L_{p}\left(Q_{j, k}\right)} \leq C 2^{-j \gamma}|u(\omega, t, \cdot)|_{W_{p}^{\gamma}\left(Q_{j, k}\right)},
$$


where the last norm is finite since $\rho_{j, k}=\operatorname{dist}\left(Q_{j, k}, \partial \mathcal{O}\right)>0$. Since $\widetilde{\psi}_{i, j, k, p^{\prime}}$ is orthogonal to every polynomial of total degree less than $\gamma$, one gets

$$
\begin{aligned}
\left|\left\langle u(\omega, t, \cdot), \widetilde{\psi}_{i, j, k, p^{\prime}}\right\rangle\right| & =\left|\left\langle u(\omega, t, \cdot)-P_{j, k}, \widetilde{\psi}_{i, j, k, p^{\prime}}\right\rangle\right| \\
& \leq\left\|u(\omega, t, \cdot)-P_{j, k}\right\|_{L_{p}\left(Q_{j, k}\right)}\left\|\widetilde{\psi}_{i, j, k, p^{\prime}}\right\|_{L_{p^{\prime}}\left(Q_{j, k}\right)} \\
& \leq C 2^{-j \gamma}|u(\omega, t, \cdot)|_{W_{p}^{\gamma}\left(Q_{j, k}\right)} \\
& \leq C 2^{-j \gamma} \rho_{j, k}^{s-\gamma}\left(\left.\left.\int_{Q_{j, k}}\left|\rho(x)^{\gamma-s}\right| D^{\gamma} u(\omega, t, x)\right|_{\ell_{p}}\right|^{p} d x\right)^{1 / p} \\
& =: C 2^{-j \gamma} \rho_{j, k}^{s-\gamma} \mu_{j, k}(\omega, t) .
\end{aligned}
$$

Fix $j \in \mathbb{N}_{0}$. Summing over all indices $(i, j, k) \in \Lambda_{j}^{0}$ and applying Hölder's inequality with exponents $\frac{p}{\tau}>1$ and $\frac{p}{p-\tau}$ one finds

$$
\begin{aligned}
\sum_{(i, j, k) \in \Lambda_{j}^{0}}\left|\left\langle u(\omega, t, \cdot), \widetilde{\psi}_{i, j, k, p^{\prime}}\right\rangle\right|^{\tau} & \leq C \sum_{(i, j, k) \in \Lambda_{j}^{0}} 2^{-j \gamma \tau} \rho_{j, k}^{(s-\gamma) \tau} \mu_{j, k}(\omega, t)^{\tau} \\
& \leq C\left(\sum_{(i, j, k) \in \Lambda_{j}^{0}} \mu_{j, k}(\omega, t)^{p}\right)^{\frac{\tau}{p}}\left(\sum_{(i, j, k) \in \Lambda_{j}^{0}} 2^{\frac{-p j \gamma \tau}{p-\tau}} \rho_{j, k}^{\frac{(s-\gamma) p \tau}{p-\tau}}\right)^{\frac{p-\tau}{p}} .
\end{aligned}
$$

Since any $x \in \mathcal{O}$ lies outside of all but at most a constant number $C>0$ of the cubes $Q_{j, k}$, $k \in \mathbb{Z}^{\mathrm{d}}$, we get the following bound for the first factor on the right hand side

$$
\begin{aligned}
\left(\sum_{(i, j, k) \in \Lambda_{j}^{0}} \mu_{j, k}(\omega, t)^{p}\right)^{\frac{\tau}{p}} & =\left(\left.\left.\sum_{(i, j, k) \in \Lambda_{j}^{0}} \int_{Q_{j, k}}\left|\rho(x)^{\gamma-s}\right| D^{\gamma} u(\omega, t, x)\right|_{\ell_{p}}\right|^{p} d x\right)^{\frac{\tau}{p}} \\
& \leq C\left\|\rho^{\gamma-s}\left|D^{\gamma} u(\omega, t, \cdot)\right|_{\ell_{p}}\right\|_{L_{p}(\mathcal{O})}^{\tau} .
\end{aligned}
$$

In order to estimate the second factor in (26) we use the Lipschitz character of the domain $\mathcal{O}$ which implies that

$$
\left|\Lambda_{j, m}\right| \leq C 2^{j(d-1)} \quad \text { for all } j, m \in \mathbb{N}_{0} .
$$

The constant $C>0$ does not depend on $j$ or $m$. Moreover, the boundedness of $\mathcal{O}$ yields $\Lambda_{j, m}=\emptyset$ for all $j, m \in \mathbb{N}_{0}$ with $m \geq C 2^{j}$. Consequently,

$$
\begin{aligned}
\left(\sum_{(i, j, k) \in \Lambda_{j}^{0}} 2^{\frac{-p j \gamma \tau}{p-\tau}} \rho_{j, k}^{\frac{(s-\gamma) p \tau}{p-\tau}}\right)^{\frac{p-\tau}{p}} & \leq\left(\sum_{m=1}^{C 2^{j}} \sum_{(i, j, k) \in \Lambda_{j, m}} 2^{\frac{-p j \gamma \tau}{p-\tau}} \rho_{j, k}^{\frac{(s-\gamma) p \tau}{p-\tau}}\right)^{\frac{p-\tau}{p}} \\
& \leq C\left(\sum_{m=1}^{C 2^{j}} 2^{j(d-1)} 2^{-j \frac{p \gamma \tau}{p-\tau}}\left(m 2^{-j}\right)^{\frac{(s-\gamma) p \tau}{p-\tau}}\right)^{\frac{p-\tau}{p}} \\
& \leq C\left(2^{j\left(d-1-\frac{s p \tau}{p-\tau}\right)}+2^{j\left(d-\frac{\gamma p \tau}{p-\tau}\right)}\right)^{\frac{p-\tau}{p}}
\end{aligned}
$$


Now, let us sum over all $j \in \mathbb{N}_{0}$ and integrate over $\Omega_{T}$ with respect to $\mathbb{P} \otimes \lambda$ on both sides of the inequality (26). By using (29) and (27) from above and Corollary 12 we get

$$
\begin{aligned}
& \int_{\Omega} \int_{0}^{T} \sum_{(i, j, k) \in \Lambda^{0}}\left|\left\langle u(\omega, t, \cdot), \widetilde{\psi}_{i, j, k, p^{\prime}}\right\rangle\right|^{\tau} d t \mathbb{P}(d \omega) \\
& \quad \leq C \sum_{j \in \mathbb{N}_{0}}\left(2^{j\left(d-1-\frac{s p \tau}{p-\tau}\right)}+2^{j\left(d-\frac{\gamma p \tau}{p-\tau}\right)}\right)^{\frac{p-\tau}{p}} \int_{\Omega} \int_{0}^{T}\left\|\rho^{\gamma-s}\left|D^{\gamma} u(\omega, t, \cdot)\right|_{\ell_{p}}\right\|_{L_{p}(\mathcal{O})}^{\tau} d t \mathbb{P}(d \omega) \\
& \quad \leq C\left(\sum_{j \in \mathbb{N}_{0}} 2^{j\left(d-1-\frac{s p \tau}{p-\tau}\right) \frac{p-\tau}{p}}+\sum_{j \in \mathbb{N}_{0}} 2^{j\left(d-\frac{\gamma p \tau}{p-\tau}\right) \frac{p-\tau}{p}}\right)\left(\|g\|_{\mathbb{H}_{p, \theta}^{\gamma-1}\left(\mathcal{O}, T ; \ell_{2}\right)}+\left\|u_{0}\right\|_{U_{p, \theta}^{\gamma}(\mathcal{O})}\right)^{\tau} .
\end{aligned}
$$

One can see that the sums on the right hand side converge if, and only if, $\alpha \in\left(0, \gamma \wedge s \frac{d}{d-1}\right)$. Finally,

$$
\int_{\Omega} \int_{0}^{T} \sum_{(i, j, k) \in \Lambda^{0}}\left|\left\langle u(\omega, t, \cdot), \widetilde{\psi}_{i, j, k, p^{\prime}}\right\rangle\right|^{\tau} d t \mathbb{P}(d \omega) \leq C\left(\|g\|_{\mathbb{H}_{p, \theta}^{\gamma-1}\left(\mathcal{O}, T ; \ell_{2}\right)}+\left\|u_{0}\right\|_{U_{p, \theta}^{\gamma}(\mathcal{O})}\right)^{\tau} .
$$

Now we estimate the term $I I$ in (25). First we fix $j \in \mathbb{N}_{0}$ and use Hölder's inequality and (28) to get

$$
\sum_{(i, j, k) \in \Lambda_{j, 0}}\left|\left\langle u(\omega, t, .), \widetilde{\psi}_{i, j, k, p^{\prime}}\right\rangle\right|^{\tau} \leq C 2^{j(d-1) \frac{p-\tau}{p}}\left(\sum_{(i, j, k) \in \Lambda_{j, 0}}\left|\left\langle u(\omega, t, .), \widetilde{\psi}_{i, j, k, p^{\prime}}\right\rangle\right|^{p}\right)^{\frac{\tau}{p}} .
$$

Summing over all $j \in \mathbb{N}_{0}$ and using Hölder's inequality again, yields

$$
\begin{aligned}
\sum_{(i, j, k) \in \Lambda \backslash \Lambda^{0}} & \left|\left\langle u(\omega, t, .), \widetilde{\psi}_{i, j, k, p^{\prime}}\right\rangle\right|^{\tau}=\sum_{j \in \mathbb{N}_{0}}\left[\sum_{(i, j, k) \in \Lambda_{j, 0}}\left|\left\langle u(\omega, t, .), \widetilde{\psi}_{i, j, k, p^{\prime}}\right\rangle\right|^{\tau}\right] \\
& \leq C \sum_{j \in \mathbb{N}_{0}}\left[2^{j(d-1) \frac{p-\tau}{p}}\left(\sum_{(i, j, k) \in \Lambda_{j, 0}}\left|\left\langle u(\omega, t, .), \widetilde{\psi}_{i, j, k, p^{\prime}}\right\rangle\right|^{p}\right)^{\frac{\tau}{p}}\right] \\
& \leq C\left(\sum_{j \in \mathbb{N}_{0}} 2^{j\left(\frac{(d-1)(p-\tau)}{p}-s p\right) \frac{p}{p-\tau}}\right)^{\frac{p-\tau}{p}}\left(\sum_{j \in \mathbb{N}_{0}} \sum_{(i, j, k) \in \Lambda_{j, 0}} 2^{j s p}\left|\left\langle u(\omega, t, .), \widetilde{\psi}_{i, j, k, p^{\prime}}\right\rangle\right|^{p}\right)^{\frac{\tau}{p}} .
\end{aligned}
$$

Using Theorem 3 and the boundedness of the extension operator, one gets for $\mathbb{P} \otimes \lambda$-almost every $(\omega, t) \in \Omega_{T}$ that

$$
\sum_{(i, j, k) \in \Lambda \backslash \Lambda^{0}}\left|\left\langle u(\omega, t, .), \widetilde{\psi}_{i, j, k, p^{\prime}}\right\rangle\right|^{\tau} \leq C\|u(\omega, t, .)\|_{B_{p, p}^{s}(\mathcal{O})}^{\tau}\left(\sum_{j \in \mathbb{N}_{0}} 2^{j\left(\frac{(d-1)(p-\tau)}{p}-s p\right) \frac{p}{p-\tau}}\right)^{\frac{p-\tau}{p}} .
$$

The series on the right hand side converges if and only if $\alpha \in\left(0, s \frac{d}{d-1}\right)$. But this is part of our assumptions, so that for $\mathbb{P} \otimes \lambda$-almost every $(\omega, t) \in \Omega_{T}$

$$
\sum_{(i, j, k) \in \Lambda \backslash \Lambda^{0}}\left|\left\langle u(\omega, t, .), \widetilde{\psi}_{i, j, k, p^{\prime}}\right\rangle\right|^{\tau} \leq C\|u(\omega, t, .)\|_{B_{p, p}^{s}(\mathcal{O})}^{\tau} .
$$


Let us integrate over $\Omega_{T}$ with respect to $\mathbb{P} \otimes \lambda$ and use Jensen's inequality to get

$$
\begin{aligned}
\int_{\Omega} \int_{0}^{T} \sum_{(i, j, k) \in \Lambda \backslash \Lambda^{0}}\left|\left\langle u(\omega, t, \cdot), \widetilde{\psi}_{i, j, k, p^{\prime}}\right\rangle\right|^{\tau} d t \mathbb{P}(d \omega) & \leq C \int_{\Omega} \int_{0}^{T}\|u(\omega, t, \cdot)\|_{B_{p, p}^{s}(\mathcal{O})}^{T} d t \mathbb{P}(d \omega) \\
& \leq C\left[\int_{\Omega} \int_{0}^{T}\|u(\omega, t, \cdot)\|_{B_{p, p}^{s}(\mathcal{O})}^{p} d t \mathbb{P}(d \omega)\right]^{\frac{\tau}{p}} .
\end{aligned}
$$

Because of (30) this proves (24). Now (23) and (22) finish the proof.

Next, we give some examples for an application of Theorem 15. We are mainly interested in the Hilbert space case $p=2$.

Example 17. Let us first consider equation (1) in the form (16) where the driving process $\left(M_{t}\right)_{t \in[0, T]}$ is a Wiener process in $\stackrel{\circ}{W}_{2}^{1}(\mathcal{O})$ with covariance operator $Q \in L_{(\text {nuc })}\left(\stackrel{\circ}{W}_{2}^{1}(\mathcal{O})\right)$. It can be represented as a stochastic integral process $\left(\int_{0}^{t} G(s) d W_{s}\right)_{t \in[0, T]}$ w.r.t. the cylindrical Wiener process $\left(W_{t}\right)_{t \in[0, T]}$ on $\ell_{2}$ by defining the integrand process $(G(t))_{t \in[0, T]}$ in the space of Hilbert-Schmidt operators $L_{(\mathrm{HS})}\left(\ell_{2}, \stackrel{\circ}{W}_{2}^{1}(\mathcal{O})\right)$ as the constant deterministic process

$$
G(\omega, t): \ell_{2} \rightarrow \stackrel{\circ}{W_{2}^{1}}(\mathcal{O}),\left(x^{\kappa}\right)_{\kappa \in \mathbb{N}} \mapsto \sum_{\kappa \in \mathbb{N}} \sqrt{\lambda_{\kappa}} x^{\kappa} e_{\kappa}, \quad(\omega, t) \in \Omega_{T},
$$

where $\left(e_{\kappa}\right)_{\kappa \in \mathbb{N}}$ is an orthonormal basis of $\stackrel{\circ}{W}_{2}^{1}(\mathcal{O})$ consisting of eigenvectors of $Q$ with positive eigenvalues $\left(\lambda_{\kappa}\right)_{\kappa \in \mathbb{N}}$.

This corresponds to defining $g=\left(g^{\kappa}\right)_{\kappa \in \mathbb{N}}$ in equation (11) by

$$
g^{\kappa}(\omega, t, \cdot):=\sqrt{\lambda_{\kappa}} e_{\kappa}, \quad \kappa \in \mathbb{N},(\omega, t) \in \Omega_{T} .
$$

It is easy to see that $g$ is an element of $\mathrm{H}_{2, d}^{1}\left(\mathcal{O}, T ; \ell_{2}\right)$. By definition

$$
\begin{aligned}
\|g\|_{\mathbb{H}_{2, d}^{1}\left(\mathcal{O}, T ; \ell_{2}\right)}^{2} & =T^{2} \sum_{n \in \mathbb{Z}} c^{n d}\left\|\zeta_{-n}\left(c^{n} \cdot\right)\left(\sqrt{\lambda_{\kappa}} e_{\kappa}\left(c^{n} \cdot\right)\right)_{\kappa \in \mathbb{N}}\right\|_{H_{2}^{1}\left(\mathbb{R}^{d} ; \ell_{2}\right)}^{2} \\
& =T^{2} \sum_{\kappa \in \mathbb{N}} \lambda_{\kappa} \sum_{n \in \mathbb{Z}} c^{n d}\left\|\zeta_{-n}\left(c^{n} \cdot\right) e_{\kappa}\left(c^{n} \cdot\right)\right\|_{H_{2}^{1}\left(\mathbb{R}^{d}\right)}^{2} \\
& =T^{2} \sum_{\kappa \in \mathbb{N}} \lambda_{\kappa}\left\|e_{\kappa}\right\|_{H_{2, d}^{1}(\mathcal{O})}^{2} .
\end{aligned}
$$

Using the norm equivalence (13), one has

$$
\begin{aligned}
\|g\|_{\mathbb{H}_{2, d}^{1}\left(\mathcal{O}, T ; \ell_{2}\right)}^{2} \leq C T^{2} \sum_{\kappa \in \mathbb{N}} \lambda_{\kappa} \sum_{|\alpha| \leq 1}\left\|\rho^{|\alpha|} D^{\alpha} e_{\kappa}\right\|_{L_{2}(\mathcal{O})}^{2} & \leq C T^{2} \sum_{\kappa \in \mathbb{N}} \lambda_{\kappa} \sum_{|\alpha| \leq 1}\left\|D^{\alpha} e_{\kappa}\right\|_{L_{2}(\mathcal{O})}^{2} \\
& =C T^{2} \sum_{\kappa \in \mathbb{N}} \lambda_{\kappa}<\infty .
\end{aligned}
$$




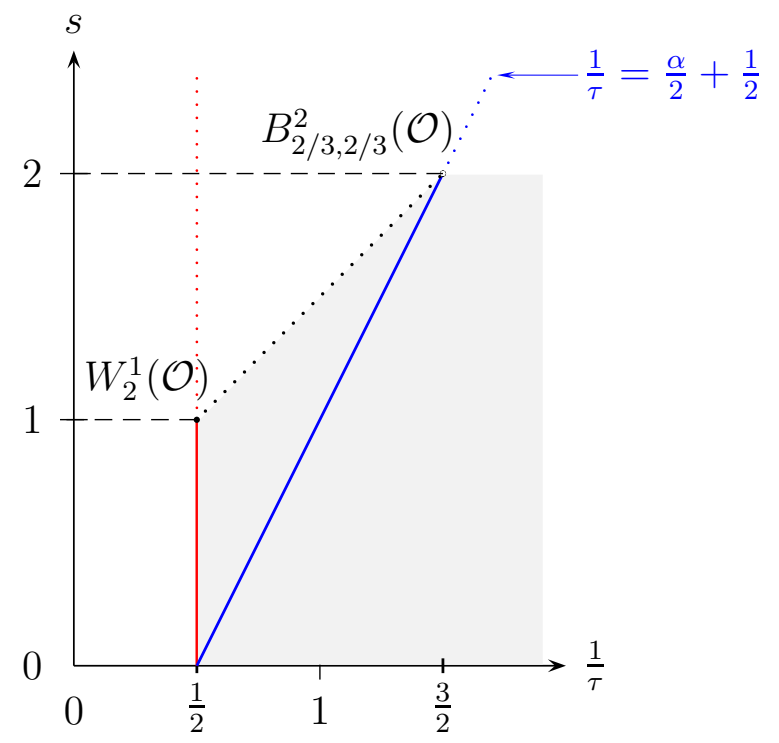

Figure 2: Besov regularity in the scale $B_{\tau, \tau}^{\alpha}(\mathcal{O}), 1 / \tau=\alpha / 2+1 / 2$, vs. Sobolev regularity of the solution, illustrated in a DEVore-TrIEBEL diagram.

Thus, in a 2-dimensional setting, Theorem 11 with $d=\theta=\gamma=2$ tells us that for every initial condition $u_{0} \in U_{2,2}^{2}(\mathcal{O})=L_{2}\left(\Omega, \mathcal{F}_{0}, \mathbb{P} ; H_{2,2}^{1}(\mathcal{O})\right)$ equation (1) has a unique solution $u$ in the class $\mathfrak{H}_{2,2}^{2}(\mathcal{O}, T) \subset \mathbb{H}_{2,0}^{2}(\mathcal{O}, T)=L_{2}\left(\Omega_{T} ; H_{2,0}^{2}(\mathcal{O})\right)$. As a trivial consequence,

$$
u \in L_{2}\left(\Omega_{T} ; W_{2}^{1}(\mathcal{O})\right)=L_{2}\left(\Omega_{T} ; B_{2,2}^{1}(\mathcal{O})\right)
$$

because we have the equality

$$
H_{2,0}^{2}(\mathcal{O})=\left\{u \in \mathcal{D}^{\prime}(\mathcal{O}): \rho^{|\alpha|-1} D^{\alpha} u \in L_{2}(\mathcal{O}) \text { for all } \alpha \in \mathbb{N}_{0}^{2} \text { with }|\alpha| \leq 2\right\} \text {. }
$$

(In fact, according to Remark 7 we even know that $u \in L_{2}\left(\Omega_{T} ; \stackrel{\circ}{W}_{2}^{1}(\mathcal{O})\right.$ ).)

Note that in general $u$ does not belong to $L_{2}\left(\Omega_{T} ; W_{2}^{s}(\mathcal{O})\right)$ for all $s<2$. Since $\mathcal{O}$ is an arbitrary bounded Lipschitz domain, certain second derivatives might explode near the boundary and the norm $\|u(\omega, t, \cdot)\|_{W_{2}^{2}(\mathcal{O})}$ as well as $\|u(\omega, t, \cdot)\|_{W_{2}^{s}(\mathcal{O})}$, where $s \in(1,2)$, might not be finite. If $\mathcal{O}$ is a polygonal domain, one can derive an explicit upper bound for the regularity in the Sobolev scale $L_{2}\left(\Omega_{T} ; W_{2}^{s}(\mathcal{O})\right), s>0$. Adapting techniques used in GRISvarD [24], 25] to our stochastic setting, one can show that $u \notin L_{2}\left(\Omega_{T} ; W_{2}^{s}(\mathcal{O})\right)$ if $s>1+\pi / \gamma_{0}$, where $\gamma_{0}$ is the measure of the largest interior angle at a corner of $\partial O$.

However, in the considered situation Theorem 15 with $s=1$ states that we have

$$
u \in L_{\tau}\left(\Omega_{T} ; B_{\tau, \tau}^{\alpha}(\mathcal{O})\right)
$$

for every $\alpha<2$ and $1 / \tau=\alpha / 2+1 / 2$. This constellation is illustrated in Figure 2, where each point $(1 / \tau, s)$ represents the smoothness spaces of functions with " $s$ derivatives in 
$L_{\tau}(\mathcal{O})$ ". Based on the knowledge that $u \in L_{2}\left(\Omega_{T} ; W_{2}^{1}(\mathcal{O})\right)$ and $u \in L_{\tau}\left(\Omega_{T} ; B_{\tau, \tau}^{\alpha}(\mathcal{O})\right)$ for all $\alpha<2,1 / \tau=\alpha / 2+1 / 2$, interpolation and embedding theorems yield that $u$ also belongs to each of the spaces $L_{\tau}\left(\Omega_{T} ; B_{\tau, \tau}^{s}(\mathcal{O})\right), 0<\tau<2, s<(1 / 2+1 / \tau) \wedge 2$. This is indicated by the shaded area.

Example 18. In view of equality (33) it is clear that we can apply Theorem 11 and Theorem [15] in the same way as in Example [17, i.e. with $d=\theta=\gamma=2$ and $s=1$, if the driving process $\left(M_{t}\right)_{t \in[0, T]}$ in (16) is a Wiener process in $W_{2}^{1}(\mathcal{O})$ with covariance operator $Q \in L_{\text {(nuc) }}\left(W_{2}^{1}(\mathcal{O})\right)$, and even if it is a Wiener process in $H_{2,2}^{1}(\mathcal{O})$ with covariance operator $Q \in L_{(\text {nuc })}\left(H_{2,2}^{1}(\mathcal{O})\right)$. In the first case $\left(M_{t}\right)_{t \in[0, T]}$ does not satisfy a zero Dirichlet boundary condition as in Example 17, and in the second case $\left(M_{t}\right)_{t \in[0, T]}$ behaves even more irregularly near the boundary in the sense that the first derivatives are allowed to blow up near $\partial \mathcal{O}$.

In these cases we choose $\left(e_{\kappa}\right)_{\kappa \in \mathbb{N}}$ in (31) and (32) to be an orthonormal basis of the space $W_{2}^{1}(\mathcal{O})$, respectively $H_{2,2}^{1}(\mathcal{O})$, consisting of eigenvectors of $Q \in L_{(\text {nuc })}\left(W_{2}^{1}(\mathcal{O})\right.$ ), respectively $Q \in L_{(\text {nuc })}\left(H_{2,2}^{1}(\mathcal{O})\right)$, with corresponding eigenvalues $\left(\lambda_{\kappa}\right)_{\kappa \in \mathbb{N}}$.

As in Example 17 the solution $u$ lies in $L_{2}\left(\Omega_{T} ; W_{2}^{1}(\mathcal{O})\right)=L_{2}\left(\Omega_{T} ; B_{2,2}^{1}(\mathcal{O})\right)$ and, by Theorem 15, it also lies in $L_{2}\left(\Omega_{T} ; B_{\tau, \tau}^{\alpha}(\mathcal{O})\right), 1 / \tau=\alpha / 2+1 / 2, \alpha<2$, see Figure 2 ,

Example 19. Let the driving process $\left(M_{t}\right)_{t \in[0, T]}$ in (16) be a time-dependent version of the stochastic wavelet expansion introduced in ABRAMOvicH et al. [1] in the context of Bayesian nonparametric regression and generalized in BochkinA [3], CiOICA et al. [7]. This noise model is formulated in terms of a wavelet basis expansion on the domain $\mathcal{O} \subset \mathbb{R}^{d}$ with random coefficients of prescribed sparsity and thus tailor-made for applying adaptive techniques with regard to the numerical approximation of the corresponding SPDEs. Via the choice of certain parameters specifying the distributions of the wavelet coefficients it also allows for an explicit control of the spatial Besov regularity of $\left(M_{t}\right)_{t \in[0, T]}$. We first describe the general noise model and then deduce a further example for the application of Theorem 15.

Let $\left\{\psi_{\lambda}: \lambda \in \nabla\right\}$ be a multiscale Riesz basis for $L_{2}(\mathcal{O})$ consisting of scaling functions at a fixed scale level $j_{0} \in \mathbb{Z}$ and of wavelets at level $j_{0}$ and all finer levels. As in the introduction, the notation we use here is different from that used in Section 2.2 because we do not consider a basis on the whole space $\mathbb{R}^{d}$ but on the bounded domain $\mathcal{O}$. Information like scale level, spatial location and type of the wavelets or scaling functions are encoded in the indices $\lambda \in \nabla$. We refer to Cohen [8, Sections 2.12, 2.13 and 3.9] and Dahmen, SCHNEIDER [15], [16], 17] for detailed descriptions of multiscale bases on bounded domains. Adopting the notation of COHEN we write $\nabla=\bigcup_{j \geq j_{0}-1} \nabla_{j}$, where for $j \geq j_{0}$ the set $\nabla_{j} \subset \nabla$ contains the indices of all wavelets $\psi_{\lambda}$ at scale level $j$ and where $\nabla_{j_{0}-1} \subset \nabla$ is the index set referring to the scaling functions at scale level $j_{0}$ which we denote by $\psi_{\lambda}, \lambda \in \nabla_{j_{0}-1}$, for the sake of notational simplicity. We make the following assumptions concerning our basis. Firstly, the cardinalities of the index sets $\nabla_{j}, j \geq j_{0}-1$, satisfy

$$
C^{-1} 2^{j d} \leq\left|\nabla_{j}\right| \leq C 2^{j d}, \quad j \geq j_{0}-1 .
$$


Secondly, we assume that the basis admits norm equivalences similar to those described in Theorem 3, There exists an $r \in \mathbb{N}$ (depending on the smoothness of the scaling functions $\psi_{\lambda}, \lambda \in \nabla_{j_{0}-1}$, and on the degree of polynomial exactness of their linear span), such that, given $p, q>0, \max \{0, d(1 / p-1)\}<s<r$, and a real valued distribution $f \in \mathcal{D}^{\prime}(\mathcal{O})$, we have $f \in B_{p, q}^{s}(\mathcal{O})$ if and only if $f$ can be represented as $f=\sum_{\lambda \in \nabla} c_{\lambda} \psi_{\lambda},\left(c_{\lambda}\right)_{\lambda \in \nabla} \subset \mathbb{R}$ (convergence in $\mathcal{D}^{\prime}(\mathcal{O})$ ), such that

$$
\left(\sum_{j=j_{0}-1}^{\infty} 2^{j q\left(s+d\left(\frac{1}{2}-\frac{1}{p}\right)\right)}\left(\sum_{\lambda \in \nabla_{j}}\left|c_{\lambda}\right|^{p}\right)^{q / p}\right)^{1 / q}<\infty .
$$

Furthermore, $\|f\|_{B_{p, q}^{s}(\mathcal{O})}$ is equivalent to the quasi-norm (35). Concrete constructions of bases satisfying these assumptions can be found in the literature mentioned above. Concerning the family of independent standard Brownian motions $\left(w_{t}^{\kappa}\right)_{t \in[0, T]}, \kappa \in \mathbb{N}$, in (1) respectively (16), we modify our notation and write $\left(w_{t}^{\lambda}\right)_{t \in[0, T]}, \lambda \in \nabla$, instead. The description of the noise model involves parameters $a \geq 0, b \in[0,1], c \in \mathbb{R}$, with $a+b>1$. For every $j \geq j_{0}-1$ we set $\sigma_{j}=\left(j-\left(j_{0}-2\right)\right)^{\frac{c d}{2}} 2^{-\frac{a\left(j-\left(j_{0}-1\right)\right) d}{2}}$ and let $Y_{\lambda}, \lambda \in \nabla_{j}$, be Bernoulli distributed random variables on $\left(\Omega, \mathcal{F}_{0}, \mathbb{P}\right)$ with parameter $p_{j}=2^{-b\left(j-\left(j_{0}-1\right)\right) d}$, such that the random variables and processes $Y_{\lambda},\left(w_{t}^{\lambda}\right)_{t \in[0, T]}, \lambda \in \nabla$, are stochastically independent. Now we are ready to define $\left(M_{t}\right)_{t \in[0, T]}$ by

$$
M_{t}:=\sum_{j=j_{0}-1}^{\infty} \sum_{\lambda \in \nabla_{j}} \sigma_{j} Y_{\lambda} \psi_{\lambda} \cdot w_{t}^{\lambda}, \quad t \in[0, T] .
$$

Using (35), (34) and $a+b>1$, it is easy to check that the infinite sum converges in $L_{2}\left(\Omega_{T} ; L_{2}(\mathcal{O})\right)$ as well as in the space $\mathcal{M}_{T}^{2, c}\left(L_{2}(\mathcal{O}),\left(\mathcal{F}_{t}\right)\right)$ of continuous, square integrable, $L_{2}(\mathcal{O})$-valued martingales w.r.t. the filtration $\left(\mathcal{F}_{t}\right)_{t \in[0, T]}$. Moreover, by the choice of the hyperparameters $a, b$ and $c$ one has an explicit control of the convergence of the infinite sum in (36) in the (quasi-) Banach spaces $L_{p_{2}}\left(\Omega_{T} ; B_{p_{1}, q}^{s}(\mathcal{O})\right), s<r, p_{1}, q>0, p_{2} \leq q$. (Compare CioicA et al. [7] which can easily be adapted to our setting.)

With regard to Theorems 11 and 15 let again $d=p=\gamma=\theta=2$. Equation (16) with $\left(M_{t}\right)_{t \in[0, T]}$ defined as above corresponds to equation (1) if we set

$$
g^{\lambda}(\omega, t, \cdot):=\sigma_{j} Y_{\lambda}(\omega) \psi_{\lambda}(\cdot), \quad \lambda \in \nabla_{j}, j \geq j_{0}-1,(\omega, t) \in \Omega_{T},
$$

and sum over all $\lambda \in \nabla$ instead of $\kappa \in \mathbb{N}$. In the following we write $\ell_{2}=\ell_{2}(\nabla)$. Since $a+b>1$ and $\|g\|_{H_{2,2}^{0}\left(\mathcal{O}, T ; \ell_{2}\right)}=\sqrt{2 / T}\|M\|_{L_{2}\left(\Omega_{T} ; L_{2}(\mathcal{O})\right)}$ we have $g \in \mathbb{H}_{2,2}^{0}\left(\mathcal{O}, T ; \ell_{2}\right)$. Let us impose a bit more smoothness on $g$ and assume that $a+b>2$. This is sufficient to ensure that $g \in \mathbb{H}_{2,2}^{1}\left(\mathcal{O}, T ; \ell_{2}\right)$ : Using (13) $)$ one sees that the $\mathbb{H}_{2,2}^{1}\left(\mathcal{O}, T ; \ell_{2}\right)$-norm of $g=\left(g^{\lambda}\right)_{\lambda \in \nabla}$ satisfies

$$
\|g\|_{\mathbb{H}_{2,2}^{1}\left(\mathcal{O}, T ; \ell_{2}\right)}^{2}=\mathbb{E} \int_{0}^{T} \sum_{n \in \mathbb{Z}} c^{n 2}\left\|\zeta_{-n}\left(c^{n} \cdot\right) g\left(t, c^{n} \cdot\right)\right\|_{H_{2}^{1}\left(\mathbb{R}^{d} ; \ell_{2}\right)}^{2} d t
$$




$$
\begin{aligned}
& =\mathbb{E} \int_{0}^{T} \sum_{\lambda \in \nabla}\left\|g^{\lambda}(t, \cdot)\right\|_{H_{2,2}^{1}(\mathcal{O})}^{2} d t \\
& =T \mathbb{E} \sum_{j=j_{0}-1}^{\infty} \sum_{\lambda \in \nabla_{j}} \sigma_{j}^{2} Y_{\lambda}^{2}\left\|\psi_{\lambda}\right\|_{H_{2,2}^{1}(\mathcal{O})}^{2} \\
& \leq C \sum_{j=j_{0}-1}^{\infty} \sum_{\lambda \in \nabla_{j}} \sigma_{j}^{2} p_{j} \sum_{|\alpha| \leq 1}\left\|\rho^{|\alpha|} D^{\alpha} \psi_{\lambda}\right\|_{L_{2}(\mathcal{O})}^{2} \\
& \leq C \sum_{j=j_{0}-1}^{\infty} \sum_{\lambda \in \nabla_{j}} \sigma_{j}^{2} p_{j}\left\|\psi_{\lambda}\right\|_{W_{2}^{1}(\mathcal{O})}^{2} .
\end{aligned}
$$

Since $W_{2}^{1}(\mathcal{O})=B_{2,2}^{1}(\mathcal{O})$ with equivalent norms we can use the equivalence (35) with $f=\psi_{\lambda}$ to get

$$
\begin{aligned}
\|g\|_{\mathbb{H}_{2,2}^{1}\left(\mathcal{O}, T ; \ell_{2}\right)}^{2} & \leq C \sum_{j=j_{0}-1}^{\infty} \sum_{\lambda \in \nabla_{j}} \sigma_{j}^{2} p_{j} 2^{2 j} \\
& =C \sum_{j=j_{0}-1}^{\infty}\left|\nabla_{j}\right|\left(j-\left(j_{0}-2\right)\right)^{2 c} 2^{-2 a(j-(j 0-1))} 2^{-2 b(j-(j 0-1))} 2^{2 j} \\
& \leq C \sum_{j=j_{0}-1}^{\infty}\left(j-\left(j_{0}-2\right)\right)^{2 c} 2^{-2 j(a+b-2)}
\end{aligned}
$$

In the last step we used (34) with $d=2$. Thus $g \in \mathbb{H}_{2,2}^{1}\left(\mathcal{O}, T ; \ell_{2}\right)$. As in Example 17 we may apply Theorems 11 and 15 to conclude that for every initial condition $u_{0} \in$ $L_{2}\left(\Omega, \mathcal{F}_{0}, \mathbb{P} ; H_{2,2}^{1}(\mathcal{O})\right)$ there exists a unique solution of equation (11) in the class $\mathfrak{H}_{2,2}^{2}(\mathcal{O}, T)$, which, in general, is not in $L_{2}\left(\Omega_{T}, W_{2}^{s}(\mathcal{O})\right)$ for all $s<2$, but it belongs to every space $L_{2}\left(\Omega_{T} ; B_{\tau, \tau}^{\alpha}(\mathcal{O})\right)$ with $\alpha<2$ and $\tau=2 /(\alpha+1)$.

Remark 20. In practice, many adaptive wavelet-based algorithms are realized with the energy norm of the problem which is equivalent to a Sobolev norm. Let us denote by $\left\{\eta_{\lambda}: \lambda \in \nabla\right\}$ a wavelet Riesz basis of $W_{2}^{s}(\mathcal{O})$ for some $s>0$, which can be obtained by rescaling the wavelet basis $\left\{\psi_{\lambda}: \lambda \in \nabla\right\}$ of $L_{2}(\mathcal{O})$, see, e.g. Cohen [8] or DAhmen [14]. For the best $N$-term approximation in this Sobolev norm, it is well known that

$$
u \in B_{\tau, \tau}^{\alpha}(\mathcal{O}), \frac{1}{\tau}=\frac{\alpha-s}{d}+\frac{1}{2} \Longrightarrow \sigma_{N, W_{2}^{s}(\mathcal{O})}(u) \leq C N^{-(\alpha-s) / d},
$$

where

$$
\sigma_{N, W_{2}^{s}(\mathcal{O})}(u):=\inf \left\{\left\|u-u_{N}\right\|_{W_{2}^{s}(\mathcal{O})}: u_{N}=\sum_{\lambda \in \Lambda} c_{\lambda} \eta_{\lambda}: \Lambda \subset \nabla,|\Lambda| \leq N, c_{\lambda} \in \mathbb{R}, \lambda \in \Lambda\right\} .
$$

Therefore, similar to the $L_{2}(\mathcal{O})$-setting, the approximation order of the best $N$-term wavelet scheme in $W_{2}^{s}(\mathcal{O})$ depends on the Besov regularity of the object one wants to approximate. 


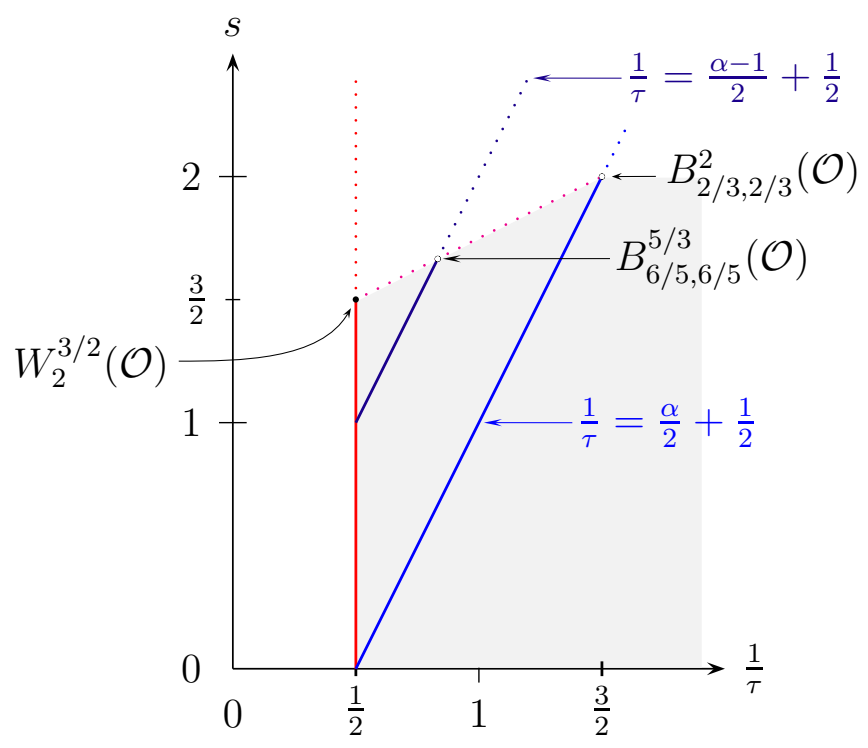

Figure 3: Besov regularity in the scale $B_{\tau, \tau}^{\alpha}(\mathcal{O}), 1 / \tau=(\alpha-1) / 2+1 / 2$, vs. Sobolev regularity of the solution illustrated in a DEVORE-TRIEBEL diagram.

There exist adaptive wavelet-based algorithms which are guaranteed to converge and which indeed asymptotically realize the convergence rate of best $N$-term approximation with respect to the Sobolev norm. For example, Cohen, Dahmen, DeVore [9] designed such an adaptive numerical scheme for solving (deterministic) elliptic PDEs. First results for parabolic problems were obtained by Schwab, Stevenson [43].

Once again, the use of adaptive algorithms is justified if the rate of approximation that can be achieved is higher than in classical uniform schemes. Let $u_{N}, N \in \mathbb{N}$, denote a uniform approximation scheme (e.g. a Galerkin approximation) of $u$. It is well-known that under certain natural conditions, see, e.g. Dahlke, Dahmen, DeVore [12] or DeVore [21] or HACKBusch [26],

$$
\left\|u-u_{N}\right\|_{W_{2}^{s}(\mathcal{O})} \leq C N^{-(\alpha-s) / d}\|u\|_{W_{2}^{\alpha}(\mathcal{O})} .
$$

This means that, even in this case, adaptivity can pay off if the Besov smoothness of the solution is higher than its Sobolev regularity.

Let us discuss this relationship in more detail for the examples from above. We consider approximation in $W_{2}^{1}(\mathcal{O})$. As already mentioned in Example 17, in general we cannot expect that the spatial Sobolev regularity of the solution is higher than $3 / 2$. Therefore, uniform schemes yield an approximation rate of $O\left(N^{-1 / 4}\right)$.

On the other hand, our main result shows that

$$
u \in L_{\tau}\left(\Omega_{T} ; B_{\tau, \tau}^{\alpha}(\mathcal{O})\right), \frac{1}{\tau}=\frac{\alpha}{2}+\frac{1}{2} \text { for all } \alpha<2 .
$$

Therefore, by interpolation and embedding of Besov spaces we can achieve that the solution is contained in all the spaces $L_{\tau}\left(\Omega_{T} ; B_{\tau, \tau}^{\alpha}(\mathcal{O})\right)$ corresponding to the points in the trapezoid 
with vertices $(1 / 2,0),(1 / 2,3 / 2),(3 / 2,2),(3 / 2,0)$ and to the points to the right of this trapezoid in the DeVore-Triebel diagram, cf. Figure 3. As a consequence, we get by a short computation, that

$$
u \in L_{\tau}\left(\Omega_{T} ; B_{\tau, \tau}^{\alpha}(\mathcal{O})\right), \frac{1}{\tau}=\frac{\alpha-1}{2}+\frac{1}{2} \text { for all } \alpha<\frac{5}{3} .
$$

Thus, best $N$-term wavelet approximation provides order $O\left(N^{-1 / 3}\right)$, so that again the use of adaptivity is completely justified.

\section{A Convergence of the stochastic integrals}

In this section we give a proof of the $\mathcal{M}_{T}^{2, c}\left(\mathbb{R},\left(\mathcal{F}_{t}\right)\right)$-convergence of the sum of the stochastic integral processes $\left(\int_{0}^{t}\left\langle g^{\kappa}(s, \cdot), \varphi\right\rangle d w_{s}^{\kappa}\right)_{t \in[0, T]}, \kappa \in \mathbb{N}$, appearing in formula (14). Let us assume that $g \in \mathbb{H}_{p, \theta}^{\gamma}\left(\mathcal{O}, T ; \ell_{2}\right)$ for some $p \in[2, \infty)$ and $\gamma, \theta \in \mathbb{R}$. We use an analogous strategy to [31, Remark 3.2. Due to the independence of the Brownian motions $\left(w_{t}^{\kappa}\right)_{t \in[0, T]}, \kappa \in \mathbb{N}$, the covariation process $\left(\left[w^{\kappa}, w^{\ell}\right]_{t}\right)_{t \in[0, T]}$ vanishes if $\kappa \neq \ell$, and we have by Itô's isometry:

$$
\begin{aligned}
\mathbb{E}\left|\sum_{\kappa=1}^{\infty} \int_{0}^{T}\left\langle g^{\kappa}(s, \cdot), \varphi\right\rangle d w_{s}^{\kappa}\right|^{2} & =\mathbb{E}\left[\sum_{\kappa=1}^{\infty} \int_{0}^{(\cdot)}\left\langle g^{\kappa}(s, \cdot), \varphi\right\rangle d w_{s}^{\kappa}, \sum_{\kappa=1}^{\infty} \int_{0}^{(\cdot)}\left\langle g^{\kappa}(s, \cdot), \varphi\right\rangle d w_{s}^{\kappa}\right]_{T} \\
& =\mathbb{E} \sum_{\kappa=1}^{\infty} \int_{0}^{T}\left|\left\langle g^{\kappa}(s, \cdot), \varphi\right\rangle\right|^{2} d s .
\end{aligned}
$$

We are going to show that the last term is less or equal a constant times $\|g\|_{\mathbb{H}_{p, \theta}^{\gamma}\left(\mathcal{O}, T ; \ell_{2}\right)}^{2}$, which is finite due to our assumption. Then the convergence of the integral processes in $\mathcal{M}_{T}^{2, c}\left(\mathbb{R},\left(\mathcal{F}_{t}\right)\right)$ follows by Doob's maximal inequality for martingales.

For $u \in \mathcal{D}^{\prime}(\mathcal{O})$ and $n \in \mathbb{Z}$ we use the notation $u_{n}:=\zeta_{-n}\left(c^{n} \cdot\right) u\left(c^{n} \cdot\right) \in \mathcal{S}^{\prime}\left(\mathbb{R}^{d}\right)$. Let us abbreviate $L_{\tau}\left(\mathbb{R}^{d}\right)$ by $L_{\tau}$ for all $\tau \geq 1$ in the sequel. Setting $p^{\prime}=p /(p-1)$, we denote by $\langle\cdot, \cdot\rangle_{L_{p} \times L_{p^{\prime}}}: L_{p} \times L_{p^{\prime}} \rightarrow \mathbb{R}$ the dual form obtained by continuous extension of $\langle\varphi, \psi\rangle=\int \varphi(x) \psi(x) d x, \varphi, \psi \in C_{0}^{\infty}\left(\mathbb{R}^{d}\right)$. Now we are ready to estimate as follows:

$$
\begin{aligned}
& \sum_{\kappa=1}^{\infty} \int_{0}^{T}\left|\left\langle g^{\kappa}(s, \cdot), \varphi\right\rangle\right|^{2} d s=\sum_{\kappa=1}^{\infty} \int_{0}^{T}\left|\sum_{n \in \mathbb{Z}} c^{n d}\left\langle g_{n}^{\kappa}(s, \cdot), \varphi_{n}\right\rangle\right|^{2} d s \\
& =\sum_{\kappa=1}^{\infty} \int_{0}^{T}\left|\sum_{n \in \mathbb{Z}} c^{n d}\left\langle(1-\Delta)^{\frac{\gamma}{2}} g_{n}^{\kappa}(s, \cdot),(1-\Delta)^{-\frac{\gamma}{2}} \varphi_{n}\right\rangle_{L_{p} \times L_{p^{\prime}}}\right|^{2} d s \\
& \leq \sum_{\kappa=1}^{\infty} \int_{0}^{T}\left[\sum _ { n \in \mathbb { Z } } c ^ { n d } | | | ( 1 - \Delta ) ^ { \frac { \gamma } { 2 } } g _ { n } ^ { \kappa } ( s , \cdot ) | \cdot | ( 1 - \Delta ) ^ { - \frac { \gamma } { 2 } } \varphi _ { n } | ^ { 1 / 2 } \left\|_{L_{2}} \cdot\left|\left\|\left|(1-\Delta)^{-\frac{\gamma}{2}} \varphi_{n}\right|^{1 / 2}\right\|_{L_{2}}\right]^{2} d s\right.\right.
\end{aligned}
$$




$$
\begin{aligned}
\leq \sum_{\kappa=1}^{\infty} \int_{0}^{T}\left[\left(\sum_{n \in \mathbb{Z}} c^{2 n d}\left\|\left|(1-\Delta)^{\frac{\gamma}{2}} g_{n}^{\kappa}(s, \cdot)\right| \cdot\left|(1-\Delta)^{-\frac{\gamma}{2}} \varphi_{n}\right|^{1 / 2}\right\|_{L_{2}}^{2}\right)^{1 / 2}\right. \\
\left.\cdot\left(\sum_{n \in \mathbb{Z}}\left\|(1-\Delta)^{-\frac{\gamma}{2}} \varphi_{n}\right\|_{L_{1}}\right)^{1 / 2}\right]^{2} d s .
\end{aligned}
$$

Here we have used Hölder's inequality twice. Since $\varphi$ has compact support in $\mathcal{O}$ and $\zeta_{-n}$ equals zero outside $\mathcal{O}_{-n}$, the functions $\varphi_{n}$ vanish on $\mathbb{R}^{d}$ for all but finitely many $n \in \mathbb{Z}$. As a consequence, the sum $\sum_{n \in \mathbb{Z}}\left\|(1-\Delta)^{-\gamma / 2} \varphi_{n}\right\|_{L_{1}}$ has only finitely many non-zero terms. Therefore,

$$
\begin{aligned}
& \sum_{\kappa=1}^{\infty} \int_{0}^{T}\left|\left\langle g^{\kappa}(s, \cdot), \varphi\right\rangle\right|^{2} d s \\
& \leq\left. C \sum_{\kappa=1}^{\infty} \int_{0}^{T} \sum_{n \in \mathbb{Z}} c^{2 n d}||(1-\Delta)^{\gamma / 2} g_{n}^{\kappa}(s, \cdot)|\cdot|(1-\Delta)^{-\gamma / 2} \varphi_{n}\right|^{1 / 2} \|_{L_{2}}^{2} d s \\
& =C \int_{0}^{T} \sum_{n \in \mathbb{Z}} c^{2 n d}\left\langle\sum_{\kappa=1}^{\infty}\left|(1-\Delta)^{\gamma / 2} g_{n}^{\kappa}(s, \cdot)\right|^{2},\left|(1-\Delta)^{-\gamma / 2} \varphi_{n}\right|\right\rangle_{L_{p / 2} \times L_{p /(p-2)}} d s
\end{aligned}
$$

where the constant $C$ depends on $\varphi$. In the last step we used the fact that

$$
(1-\Delta)^{\gamma / 2} g_{n}(s, \cdot)=\left((1-\Delta)^{\gamma / 2} g_{n}^{\kappa}(s, \cdot)\right)_{\kappa \in \mathbb{N}} \in L_{p}\left(\mathbb{R}^{d} ; \ell_{2}\right)
$$

$\mathbb{P} \otimes \lambda$-almost everywhere in $\Omega_{T}$, which results from $g$ being an element of $\mathbb{H}_{p, \theta}^{\gamma}\left(\mathcal{O} ; \ell_{2}\right)$. Applying again Hölder's inequality we obtain

$$
\begin{aligned}
& \sum_{n \in \mathbb{Z}} c^{2 n d}\left\langle\sum_{\kappa=1}^{\infty}\left|(1-\Delta)^{\gamma / 2} g_{n}^{\kappa}(s, \cdot)\right|^{2},\left|(1-\Delta)^{-\gamma / 2} \varphi_{n}\right|\right\rangle_{L_{p / 2} \times L_{p /(p-2)}} \\
& \leq \sum_{n \in \mathbb{Z}} c^{2 n \theta}\left\|\left|(1-\Delta)^{\gamma / 2} g_{n}(s, \cdot)\right|_{\ell_{2}}\right\|_{L_{p}}^{1 / 2} c^{2 n(d-\theta)}\left\|(1-\Delta)^{-\gamma / 2} \varphi_{n}\right\|_{L_{p /(p-2)}} \\
& \leq C\left(\sum_{n \in \mathbb{Z}} c^{n \theta}\left\|\left|(1-\Delta)^{\gamma / 2} g_{n}(s, \cdot)\right|_{\ell_{2}}\right\|_{L_{p}}^{p}\right)^{\frac{2}{p}}\left(\sum_{n \in \mathbb{Z}} c^{2 n(d-\theta) p /(p-2)}\left\|(1-\Delta)^{-\gamma / 2} \varphi_{n}\right\|_{L_{p /(p-2)} /(p-2)}^{p}\right)^{\frac{p-2}{p}} \\
& \leq C\left(\sum_{n \in \mathbb{Z}} c^{n \theta}\left\|\left|(1-\Delta)^{\gamma / 2} g_{n}(s, \cdot)\right|_{\ell_{2}}\right\|_{L_{p}}^{p}\right)^{\frac{2}{p}}
\end{aligned}
$$

where we have used that $p \leq 2$ and that only finitely many of the $\varphi_{n}, n \in \mathbb{Z}$, are non-zero. All in all we have shown

$$
\sum_{\kappa=1}^{\infty} \int_{0}^{T}\left|\left\langle g^{\kappa}(s, \cdot), \varphi\right\rangle\right|^{2} d s \leq C \int_{0}^{T}\left(\sum_{n \in \mathbb{Z}} c^{n \theta}\left\|\left|(1-\Delta)^{\gamma / 2} g_{n}(s, \cdot)\right|_{\ell_{2}}\right\|_{L_{p}}^{p}\right)^{\frac{2}{p}} d s .
$$


Finally, taking the expectation and applying Jensen's inequality yields

$$
\mathbb{E} \sum_{\kappa=1}^{\infty} \int_{0}^{T}\left|\left\langle g^{\kappa}(s, \cdot), \varphi\right\rangle\right|^{2} d s \leq C\|g\|_{\mathbb{H}_{p, \theta}^{\gamma}\left(\mathcal{O}, T ; \ell_{2}\right)}^{2}
$$

and this finishes the proof.

\section{B General linear equations}

In the introduction we have indicated that our main result can be extended to equation (3). The major reason is, that by a result from KIM [30] an estimate similar to the one proved in Corollary 12 holds not only for the model equation (1) but for equations of the type (3), provided the coefficients $a^{\mu \nu}, b^{\mu}, c, \sigma^{\mu \kappa}$ and $\eta^{\kappa}$, the free terms $f$ and $g^{\kappa}$ and the initial value $u_{0}$ fulfil certain conditions. We can use this fact to extend our regularity result to such equations. In this section we want to get more precise and point out how to do this.

For the convenience of the reader we begin by presenting the result from KIM [30, Theorem 2.8]. Therefore, we need some additional notations. For $x, y \in \mathcal{O}$ we shall write $\rho(x, y):=\rho(x) \wedge \rho(y)$. For $\alpha \in \mathbb{R}, \delta \in(0,1]$ and $k \in \mathbb{N}_{0}$ we set:

$$
\begin{aligned}
{[f]_{k}^{(\alpha)}:=\sup _{x \in \mathcal{O}} \rho^{k+\alpha}(x)\left|D^{k} f(x)\right| \quad \text { and } \quad[f]_{k+\delta}^{(\alpha)}:=\sup _{\substack{x, y \in \mathcal{O} \\
|\beta|=k}} \rho^{k+\alpha}(x, y) \frac{\left|D^{\beta} f(x)-D^{\beta} f(y)\right|}{|x-y|^{\delta}} } \\
|f|_{k}^{(\alpha)}:=\sum_{j=0}^{k}[f]_{j}^{(\alpha)} \quad \text { and } \quad|f|_{k+\delta}^{(\alpha)}:=|f|_{k}^{(\alpha)}+[f]_{k+\delta}^{(\alpha)},
\end{aligned}
$$

whenever it makes sense. We shall use the same notations for $\ell_{2}$-valued functions (just replace the absolute values in the above definitions by the $\ell_{2}$-norms). Furthermore, let's fix an arbitrary function

$$
\mu_{0}:[0, \infty) \rightarrow[0, \infty),
$$

vanishing only on the set of nonnegative integers, i.e. $\mu_{0}(j)=0$ if and only if $j \in \mathbb{N}_{0}$. We set

$$
t_{+}:=t+\mu_{0}(t)
$$

Now we are able to present the assumptions on the coefficients of equation (3) (see KIM [30, Assumptions 2.5 and 2.6]).

[K1] For any fixed $x \in \mathcal{O}$, the coefficients

$$
a^{\mu \nu}(., ., x), b^{\mu}(., ., x), c(., ., x), \sigma^{\mu \kappa}(., ., x), \eta^{\kappa}(., ., x): \Omega \times[0, T] \rightarrow \mathbb{R}
$$

are predictable processes with respect to the given normal filtration $\left(\mathcal{F}_{t}\right)_{t \in[0, T]}$. 
[K2] (Stochastic parabolicity) There are constants $\delta_{0}, K>0$, such that for all $(\omega, t, x) \in$ $\Omega \times[0, T] \times \mathcal{O}$ and $\lambda \in \mathbb{R}^{d}:$

$$
\delta_{0}|\lambda|^{2} \leq \overline{a^{\mu \nu}}(\omega, t, x) \lambda_{\mu} \lambda_{\nu} \leq K|\lambda|^{2},
$$

where $\overline{a^{\mu \nu}}:=a^{\mu \nu}-\frac{1}{2}\left\langle\sigma^{\mu}, \sigma^{\nu}\right\rangle_{\ell_{2}}$ for $\mu, \nu \in\{1, \ldots, d\}$.

[K3] For all $(\omega, t) \in \Omega \times[0, T]$ :

$$
\begin{aligned}
\left|a^{\mu \nu}(\omega, t, .)\right|_{|\gamma|_{+}}^{(0)}+\left|b^{\mu}(\omega, t, .)\right|_{|\gamma|_{+}}^{(1)} & +|c(\omega, t, .)|_{|\gamma|_{+}}^{(2)} \\
& +\left|\sigma^{\mu}(\omega, t, .)\right|_{|\gamma|_{+}}^{(0)}+|\nu(\omega, t, .)|_{|\gamma+1|_{+}}^{(1)} \leq K .
\end{aligned}
$$

[K4] The coefficients $a^{\mu \nu}$ and $\sigma^{\mu}$ are uniformly continuous in $x \in \mathcal{O}$, i.e. for any $\epsilon>0$ there is a $\delta=\delta(\epsilon)>0$, such that

$$
\left|a^{\mu \nu}(\omega, t, x)-a^{\mu \nu}(\omega, t, y)\right|+\left|\sigma^{\mu}(\omega, t, x)-\sigma^{\mu}(\omega, t, y)\right|_{\ell_{2}} \leq \epsilon,
$$

for all $(\omega, t) \in \Omega \times[0, T]$, whenever $x, y \in \mathcal{O}$ with $|x-y| \leq \delta$.

[K5] The behaviour of the coefficients $b^{\mu}, c$ and $\nu$ can be controlled near the boundary of $\mathcal{O}$ in the following way:

$$
\lim _{\substack{\rho(x) \rightarrow 0 \\ x \in \mathcal{O}}} \sup _{\substack{\omega \in \Omega \\ t \in[0, T]}}\left\{\rho(x)\left|b^{\mu}(\omega, t, x)\right|+\rho^{2}(x)|c(\omega, t, x)|+|\nu(\omega, t, x)|_{\ell_{2}}\right\}=0 .
$$

Here is the main result of KIM [30].

Theorem 21. Let $p \in[2, \infty)$ and let assumptions $[\mathrm{K} 1]-[\mathrm{K} 5]$ be satisfied with $K, \delta_{0}>0$. Then there exists a constant $\kappa_{0}=\kappa_{0}\left(d, p, \delta_{0}, K, \mathcal{O}\right) \in(0,1)$ such that, if $\theta \in\left(d-\kappa_{0}, d+\right.$ $\left.\kappa_{0}+p-2\right)$, for any $f \in \mathbb{H}_{p, \theta+p}^{\gamma-2}(\mathcal{O}, T), g \in \mathbb{H}_{p, \theta}^{\gamma-1}\left(\mathcal{O}, T ; \ell_{2}\right)$ and $u_{0} \in U_{p, \theta}^{\gamma}(\mathcal{O})$, equation (3) with initial value $u_{0}$ admits a unique solution $u \in \mathfrak{H}_{p, \theta}^{\gamma}(\mathcal{O}, T)$, i.e., there exists an (up to indistinguishability) unique $\mathcal{D}^{\prime}(\mathcal{O})$-valued predictable process $u \in \mathbb{H}_{p, \theta-p}^{\gamma}(\mathcal{O}, T)$, such that for any $\varphi \in C_{0}^{\infty}(\mathcal{O})$ the equality

$$
\begin{aligned}
\langle u(t, .), \varphi\rangle= & \langle u(0, .), \varphi\rangle \\
& +\int_{0}^{t}\left\langle a^{\mu \nu}(s, .) u_{x_{\mu} x_{\nu}}(s, .)+b^{\mu}(s, .) u_{x_{\mu}}(s, .)+c(s, .) u(s, .)+f(s, .), \varphi\right\rangle \mathrm{d} s \\
& +\sum_{\kappa=1}^{\infty} \int_{0}^{t}\left\langle\sigma^{\mu \kappa}(s, .) u_{x_{\mu}}(s, .)+\eta^{\kappa}(s, .) u(s, .)+g^{\kappa}(s, .), \varphi\right\rangle \mathrm{d} w_{s}^{\kappa}
\end{aligned}
$$

holds for all $t \in[0, T]$ with probability 1 . Moreover, for this solutions we have

$$
\|u\|_{\mathfrak{H}_{p, \theta}^{\gamma}(\mathcal{O}, T)}^{p} \leq C\left(\|f\|_{\mathbb{H}_{p, \theta+p}^{\gamma-2}(\mathcal{O}, T)}^{p}+\|g\|_{\mathbb{H}_{p, \theta}^{\gamma-1}\left(\mathcal{O}, T ; \ell_{2}\right)}^{p}+\left\|u_{0}\right\|_{U_{p, \theta}^{\gamma}(\mathcal{O})}^{p}\right)
$$

where $C$ is a constant depending only on $d, \gamma, p, \theta, \delta_{0}, K, T$ and the domain $\mathcal{O}$. 
An immediate consequence of this theorem is the following estimate.

Corollary 22. In the situation of Theorem 21 with $\gamma=m \in \mathbb{N}$, the following inequality holds for every $\tau \in[0, p]$ :

$$
\begin{aligned}
\int_{\Omega} \int_{0}^{T}\left\|\rho^{m-\beta}\left|D^{m} u(\omega, t, \cdot)\right|_{\ell_{p}}\right\|_{L_{p}(\mathcal{O})}^{\tau} d t & \mathbb{P}(d \omega) \\
& \leq C\left(\|f\|_{\mathbb{H}_{p, \theta+p}^{m-2}}+\|g\|_{\mathbb{H}_{p, \theta}^{m-1}\left(\mathcal{O}, T ; \ell_{2}\right)}+\left\|u_{0}\right\|_{U_{p, \theta}^{m}(\mathcal{O})}\right)^{\tau}
\end{aligned}
$$

where $\beta=1+\frac{d-\theta}{p}$.

Proof. Just repeat the arguments of the proof of Corollary 12 and use estimate (37) instead of (15) at the beginning.

Now we can present our main result in the generalized setting.

Theorem 23. Let $p \in[2, \infty)$ and let assumptions $[\mathrm{K} 1]-[\mathrm{K} 5]$ be satisfied with appropriate constants $K, \delta_{0}>0$. Moreover, let $f \in \mathbb{H}_{p, \theta+p}^{\gamma-2}(\mathcal{O}, T), g \in \mathbb{H}_{p, \theta}^{\gamma-1}\left(\mathcal{O}, T ; \ell_{2}\right)$ and $u_{0} \in U_{p, \theta}^{\gamma}(\mathcal{O})$ for some $\gamma \in \mathbb{N}$. Denote by $u$ the unique solution of equation (3) in the class $\mathfrak{H}_{p, \theta}^{\gamma}(\mathcal{O}, T)$ for a given $\theta \in\left(d-\kappa_{0}, d-2+p+\kappa_{0}\right)$, where $\kappa_{0}=\kappa_{0}\left(d, p, \delta_{0}, K, \mathcal{O}\right) \in(0,1)$ as in Theorem 21. Assume furthermore that $u \in L_{p}\left(\Omega_{T} ; B_{p, p}^{s}(\mathcal{O})\right)$ for some $s \in\left(0, \gamma \wedge\left(1+\frac{d-\theta}{p}\right)\right]$.

Then, we have

$$
u \in L_{\tau}\left(\Omega_{T} ; B_{\tau, \tau}^{\alpha}(\mathcal{O})\right), \quad \frac{1}{\tau}=\frac{\alpha}{d}+\frac{1}{p}, \quad \text { for all } \quad \alpha \in\left(0, \gamma \wedge \frac{s d}{d-1}\right),
$$

and the following estimation holds

$$
\|u\|_{L_{\tau}\left(\Omega_{T} ; B_{\tau, \tau}^{\alpha}(\mathcal{O})\right)} \leq C\left(\|f\|_{\mathbb{H}_{p, \theta+p}^{\gamma-2}(\mathcal{O}, T)}+\|g\|_{\mathbb{H}_{p, \theta}^{\gamma-1}\left(\mathcal{O}, T ; \ell_{2}\right)}+\left\|u_{0}\right\|_{U_{p, \theta}^{\gamma}(\mathcal{O})}+\|u\|_{L_{p}\left(\Omega_{T} ; B_{p, p}^{s}(\mathcal{O})\right)}\right) .
$$

Proof. We can argue like we did in the proof of Theorem 15, We just have to use Corollary 22 where we used Corollary 12.

\section{References}

[1] F. Abramovich, T. Sapatinas, B.W. Silverman, Wavelet threshholding via a Bayesian approach, J. R. Stat. Soc., Ser. B, Stat. Methodol. 60 (1998) 725-749.

[2] R.A. Adams, J.J.F. Fournier, Sobolev Spaces, second ed., Elsevier, 2003.

[3] N. Bochkina, Besov regularity of functions with sparse random wavelet coefficients, Manuscript (2006) URL: http://www.bgx.org.uk/Natalia/Bochkina_BesovWavelets.pdf, last accessed: 05/11/2010.

[4] Z. Brzeźniak, Stochastic Partial Differential Equations in M-Type 2 Banach Spaces, Potential Anal. 4 (1995) 1-45. 
[5] Z. Brzeźniak, On Stochastic Convolutions in Banach Spaces and Applications, Stochastics Stochastics Rep. 61 (1997) 245-295.

[6] Z. Brzeźniak, J.M.A.M. van Neerven, Stochastic convolution in separable Banach spaces and the stochastic linear Cauchy problem, Stud. Math. 143 (2000) 43-74.

[7] P.A. Cioica, S. Dahlke, N. Döhring, S. Kinzel, F. Lindner, T. Raasch, K. Ritter, R.L. Schilling, Adaptive Wavelet Methods for Elliptic Stochastic Partial Differential Equations, In preparation.

[8] A. Cohen, Numerical Analysis of Wavelet Methods, Elsevier, 2003.

[9] A. Cohen, W. Dahmen, R.A. DeVore, Adaptive wavelet methods for elliptic operator equations: Convergence rates, Math. Comput. 70 (2001) 27-75.

[10] A. Cohen, W. Dahmen, R.A. DeVore, Adaptive Wavelet Methods II-Beyond the Elliptic Case, Found. Comput. Math. 2 (2002) 203-245.

[11] A. Cohen, I. Daubechies, J.-C. Feauveau, Biorthogonal Bases of Compactly Supported Wavelets, Commun. Pure Appl. Math. 45 (1992) 485-560.

[12] S. Dahlke, W. Dahmen, R.A. DeVore, Nonlinear Approximation and Adaptive Techniques for Solving Elliptic Operator Equations, in: W. Dahmen, A. Kurdila, P. Oswald (Eds.), Multiscale Wavelet Methods for Partial Differential Equations, Academic Press, 1997, pp. 237-283.

[13] S. Dahlke, R.A. DeVore, Besov regularity for elliptic boundary value problems, Commun. Partial Differ. Equations 22 (1997) 1-16.

[14] W. Dahmen, Wavelet and Multiscale Methods for Operator Equations, Acta Numerica 6 (1997) 55228.

[15] W. Dahmen, R. Schneider, Wavelets with Complementary Boundary Conditions - Function Spaces on the Cube, Result. Math. 34 (1998) 255-293.

[16] W. Dahmen, R. Schneider, Composite wavelet bases for operator equations, Math. Comput. 68 (1999) $1533-1567$.

[17] W. Dahmen, R. Schneider, Wavelets on Manifolds I: Construction and Domain Decomposition, SIAM J. Math. Anal. 31 (1999) 184-230.

[18] G. Da Prato, J. Zabczyk, Stochastic Equations in Infinite Dimensions, Cambridge University Press, 1992.

[19] I. Daubechies, Ten Lectures on Wavelets, SIAM, 1992.

[20] J. Dettweiler, J.M.A.M van Neerven, L. Weis, Space-Time Regularity of Solutions of the Parabolic Stochastic Cauchy Problem, Stochastic Anal. Appl. 24 (2006) 843-869.

[21] R.A. DeVore, Nonlinear Approximation, Acta Numerica 7 (1998) 51-150.

[22] R.A. DeVore, R. Sharpley, Maximal functions measuring smoothness, Mem. Am. Math. Soc. 293 (1994) $1-115$.

[23] S. Dispa, Intrinsic characterizations of Besov spaces on Lipschitz domains, Math. Nachr. 260 (2003) 21-33.

[24] P. Grisvard, Elliptic Problems in Nonsmooth Domains, Pitman, 1985.

[25] P. Grisvard, Singularities in Boundary Value Problems, Springer, 1992.

[26] W. Hackbusch, Elliptic differential equations: theory and numerical treatment, Springer, 1992.

[27] L. Hörmander, The analysis of linear partial differential operators. I. Distribution theory and Fourier analysis, second ed., Springer, 1990.

[28] D. Jerison, C.E. Kenig, The Inhomogeneous Dirichlet Problem in Lipschitz Domains, J. Funct. Anal. 130 (1995) 161-219.

[29] K.-H. Kim, On stochastic partial differential equations with variable coefficients in $C^{1}$ domains, Stochastic Process. Appl. 112 (2004) 261-283.

[30] K.-H. Kim, An $L_{p}$-Theory of SPDEs on Lipschitz Domains, Potential Anal. 29 (2008) 303-326.

[31] N.V. Krylov, An Analytic Approach to SPDEs, in: B.L. Rozovskii, R. Carmona (Eds.), Stochastic Partial Differential Equations. Six Perspectives, American Mathematical Society, 1999, pp. 185-242.

[32] N.V. Krylov, S.V. Lototsky, A Sobolev Space Theory of SPDE with Constant Coefficients on a Half Line, SIAM J. Math. Anal. 30 (1999) 298-325. 
[33] A. Kufner, Weighted Sobolev Spaces, Teubner, 1980.

[34] G. Kyriazis, Wavelet Coefficients Measuring Smoothness in $H_{p}\left(\mathbb{R}^{d}\right)$, Appl. Comput. Harmon. Anal. 3 (1996) 100-119.

[35] S.V. Lototsky, Sobolev Spaces with Weights in Domains and Boundary Value Problems for Degenerate Elliptic Equations, Methods Appl. Anal. 7 (2000) 195-204.

[36] Y. Meyer, Wavelets and operators, Cambridge University Press, 1992.

[37] J.M.A.M. van Neerven, L. Weis, Stochastic integration of functions with values in a Banach space, Stud. Math. 166 (2005) 131-170.

[38] J.M.A.M. van Neerven, M.C. Veraar, L. Weis, Stochastic integration in UMD Banach spaces, Ann. Probab. 35 (2007) 1438-1478.

[39] J.M.A.M. van Neerven, M.C. Veraar, L. Weis, Stochastic evolution equations in UMD Banach spaces, J. Funct. Anal. 255 (2008) 940-993.

[40] S. Peszat, J. Zabczyk, Stochastic partial differential equations with Lévy noise. An evolution equation approach, Cambridge University Press, 2007.

[41] V.S. Rychkov, On Restrictions and Extensions of the Besov and Triebel-Lizorkin Spaces with Respect to Lipschitz Domains, J. Lond. Math. Soc. 60 (1999) 237-257.

[42] R.L. Schilling, Measures, Integrals and Martingales, Cambridge University Press, 2005.

[43] C. Schwab, R. Stevenson, Space-time adaptive wavelet methods for parabolic evolution problems, Math. Comp. 78 (2009) 1293-1318.

[44] H. Triebel, Interpolation Theory, Function Spaces, Differential Operators, second ed., Barth, 1995.

[45] H. Triebel, Theory of Function Spaces III, Birkhäuser, 2006.

[46] A. Pietsch, Operator Ideals, VEB Deutscher Verlag der Wissenschaften, 1978.

Petru A. Cioica, Stephan Dahlke, Stefan Kinzel: Philipps-Universität Marburg, FB Mathematik und Informatik, AG Numerik/Optimierung, Hans-Meerwein-Straße, 35032 Marburg, Germany.

\{cioica, dahlke, kinzel\}@mathematik.uni-marburg.de

Felix Lindner, René L. Schilling: TU Dresden, FB Mathematik, Institut für Mathematische Stochastik, Zellescher Weg 12-14, 01069 Dresden, Germany. \{felix.lindner, rene.schilling\}@tu-dresden.de

Thorsten Raasch: Johannes-Gutenberg-Universität Mainz, Institut für Mathematik, AG Numerische Mathematik, Staudingerweg 9, 55099 Mainz, Germany. raasch@uni-mainz.de

Klaus Ritter: TU Kaiserslautern, Department of Mathematics, Computational Stochastics Group, ErwinSchrödinger-Straße, 67663 Kaiserslautern, Germany. ritter@mathematik.uni-kl.de 